\title{
Electroweak restoration at the LHC and beyond: The $V h$ channel
}

\author{
Li Huang $\odot,{ }^{1, *}$ Samuel D. Lane, ${ }^{1,2, \dagger}$ Ian M. Lewis $\odot,{ }^{1, \hbar}$ and Zhen Liu $\oplus^{3,4, \S}$ \\ ${ }^{1}$ Department of Physics and Astronomy, University of Kansas, Lawrence, Kansas 66045 USA \\ ${ }^{2}$ Department of Physics, Brookhaven National Laboratory, Upton, New York 11973 USA \\ ${ }^{3}$ Maryland Center for Fundamental Physics, Department of Physics, University of Maryland, \\ College Park, Maryland, 20742 USA \\ ${ }^{4}$ School of Physics and Astronomy, University of Minnesota, Minneapolis, Minnesota, 55455 USA
}

(Received 8 December 2020; accepted 23 February 2021; published 22 March 2021)

\begin{abstract}
The LHC is exploring electroweak (EW) physics at the scale EW symmetry is broken. As the LHC and new high energy colliders push our understanding of the Standard Model to ever-higher energies, it will be possible to probe not only the breaking of but also the restoration of EW symmetry. We propose to observe EW restoration in double EW boson production via the convergence of the Goldstone boson equivalence theorem. This convergence is most easily measured in the vector boson plus Higgs production, $V h$, which is dominated by the longitudinal polarizations. We define EW restoration by carefully taking the limit of zero Higgs vacuum expectation value (vev). EW restoration is then measured through the ratio of the $p_{T}^{h}$ distributions between $V h$ production in the Standard Model and Goldstone boson plus Higgs production in the zero vev theory, where $p_{T}^{h}$ is the Higgs transverse momentum. As EW symmetry is restored, this ratio converges to one at high energy. We present a method to extract this ratio from collider data. With a full signal and background analysis, we demonstrate that the $14 \mathrm{TeV}$ HL-LHC can confirm that this ratio converges to one to $40 \%$ precision while at the $27 \mathrm{TeV}$ HE-LHC the precision will be $6 \%$. We also investigate statistical tests to quantify the convergence at high energies. Our analysis provides a roadmap for how to stress test the Goldstone boson equivalence theorem and our understanding of spontaneously broken symmetries, in addition to confirming the restoration of EW symmetry.
\end{abstract}

DOI: 10.1103/PhysRevD.103.053007

\section{INTRODUCTION}

With the discovery of a Standard Model-like Higgs boson $[1,2]$, we entered a new era of probing the nature of electroweak (EW) symmetry breaking. Through the measurement of many EW and Higgs boson processes, the LHC is exploring the nature of a spontaneously broken symmetry at and above its breaking scale. As the LHC continues to gather data, it pushes these precision measurements and our understanding of EW symmetry breaking (EWSB) to ever-higher energies. These higher energies are very interesting for precision EW measurements [3-40], in particular at future high energy colliders. As we get further above the EW scale, EW particles are essentially massless, and new interesting SM physics begins to appear. For example, the

\footnotetext{
*huangli@ku.edu

†samuel.lane@ku.edu

*ian.lewis@ku.edu

§liuphys@umd.edu
}

Published by the American Physical Society under the terms of the Creative Commons Attribution 4.0 International license. Further distribution of this work must maintain attribution to the author(s) and the published article's title, journal citation, and DOI. Funded by SCOAP ${ }^{3}$. massive EW gauge bosons become partons and must be included in parton distribution functions as EW multiplets [41-46] and parton showers [26,47-49]. While these effects are intrinsically interesting and necessary to our understanding of the SM, they have also been shown to significantly impact searches for beyond the SM physics [50].

In this paper, we propose a new study to test one of the central behaviors of the SM at high energy: restoration of EW symmetry. We will propose a systematic analysis to observe this restoration at high energy colliders. Our study will open new analysis methods to stress test our understanding of the SM and the spontaneous breaking of EW theory. The main ingredient of our analysis is that as massive bosons become massless, their longitudinal modes can be replaced by the associated Goldstone bosons via the Goldstone boson equivalence theorem (GBET). Indeed, the GBET is a central ingredient to our understanding of the quantum field theory of spontaneously broken symmetries. Hence, our analysis provides a roadmap for how to empirically test the GBET, deepen our knowledge of spontaneously broken symmetries, and confirm the restoration of EW symmetry at high energies.

In the SM, restoration of EW symmetry is equivalent to taking the limit where the Higgs vacuum expection value 
(vev), $v$, goes to zero. ${ }^{1}$ In this limit the EW gauge bosons become massless. That is, only the transverse polarizations persist and the longitudinal polarizations are replaced by their associated Goldstone bosons, i.e., the GBET mentioned above. There is a long history [51-64] of trying to observe the GBET via longitudinal vector boson scattering. One of the interesting things about longitudinal vector boson scattering is that this process probes the quartic Goldstone boson coupling, which arises via the Higgs potential:

$$
\begin{aligned}
V(H)= & -\mu^{2} H^{\dagger} H+\lambda\left(H^{\dagger} H\right)^{2} \\
& \text { where } H=\left(\begin{array}{c}
G^{+} \\
\frac{1}{\sqrt{2}}\left(v+h+i G^{0}\right)
\end{array}\right)
\end{aligned}
$$

is the Higgs doublet, $G^{+}, G^{0}$ are the Goldstone bosons, $h$ is the Higgs boson, and $v=246 \mathrm{GeV}$ is the Higgs vev. Hence, longitudinal vector boson scattering probes the shape of the Higgs potential and the source of EWSB. Additionally, this process violated perturbative unitarity without a Higgs boson [52-54]. However, with the observation of a light Higgs boson with SM-like couplings to EW gauge bosons [65-68], longitudinal vector boson scattering is effectively unitarized with the violation of perturbative unitarity pushed to multi$\mathrm{TeV}$ energies [53,64,69-73], making it difficult to observe.

As the above makes clear, the observation of EW symmetry restoration and the GBET is simplest in processes that are dominated by longitudinally polarized gauge bosons. Such a process is Higgs production in association with an EW gauge boson: $q \bar{q}^{\prime} \rightarrow V h$ with $V=$ $W^{ \pm}, Z(V h)$. In the GBET, the $q \bar{q}^{\prime} \rightarrow V h$ production is equivalent to $q \bar{q}^{\prime} \rightarrow G^{ \pm, 0} h$ production $(G h)$ which arises from the Higgs kinetic term:

$$
\mathcal{L}_{\text {kin }}=\left|D_{\mu} H\right|^{2} .
$$

The kinetic term contains the trilinear interactions (a) $Z-G^{0}-h, W^{ \pm}-G^{\mp}-h$ and (b) $Z / \gamma-G^{+}-G^{-}$, $W^{ \pm}-G^{\mp}-G^{0}$. The interactions (a) contribute to the processes $q \bar{q} \rightarrow V_{L} h$, where the subscript $L$ indicates a longitudinally polarized vector boson. The interactions (b) contribute to pair production of longitudinally polarized gauge bosons $q \bar{q} \rightarrow V_{L} V_{L}^{\prime}$, where $V^{\prime}=Z, W^{ \pm}$. However, the pair production of gauge bosons $q \bar{q}^{\prime} \rightarrow V V^{\prime}$ is dominated by transverse polarizations to high energy $[11,74,75]$. For the $V h$ channel, the contribution from transversely polarized vector bosons is suppressed since a portion of the Higgs doublet already exists in the final state.

From this discussion, Higgs production in association with $W^{ \pm}$or $Z$ is a prime candidate to observe EW restoration. In this paper we present an analysis strategy to do precisely this. While this may seem straightforward, complications immediately arise when trying to observe

\footnotetext{
${ }^{1}$ In a consistent manner.
}

EW restoration at hadron colliders. Namely, the vector and Higgs bosons are intermediate states, and the collider observes their decay products. These decays occur at the EW scale and the GBET is not valid. This is clear by noting that while vector boson couplings to fermions are universal across generations, the Goldstone bosons couple like mass. Hence, their branching ratios are vastly different and it is necessary to unfold to the underlying two-to-two process.

As a proof of principle, we show that the convergence of the EW restoration can be observed in $q \bar{q}^{\prime} \rightarrow V h$ in the Higgs transverse momentum distribution at the $14 \mathrm{TeV}$ high luminosity LHC (HL-LHC) and the proposed $27 \mathrm{TeV}$ high energy LHC (HE-LHC). We will define a signal strength that is a ratio of the vector boson $V h$ and Goldstone boson $G h$ processes. In fact, numerically the signal strength is the same for both $W^{ \pm} h$ and $Z h$ production. At high Higgs transverse momentum, we show that it is possible to observe the convergence of this signal strength to one, indicating that EW symmetry is restored. We will also explore various test statistics to determine how well the GBET converges. In particular, we propose a modified the Kullback-Leibler divergence to quantify the convergence.

In Sec. II we give the theoretical foundation for our work. Helicity amplitudes of di-boson processes and polarized production rates are given in Sec. II(a), and in Sec. II(b) we define what we mean by the $v \rightarrow 0$ limit. In Sec. III we define a likelihood to perform the unfolding, and define the relevant signal strength. We present our collider analysis in Sec. IV, which is based on a deep neural network (DNN). In Sec. V, we present our results showing the convergence of our signal strength as well as the modified Kullback-Leibler divergence. Finally, in Sec. VI we conclude.

\section{THEORY}

\section{A. Amplitudes}

To observe the convergence of the Goldstone boson equivalence theorem and restoration of EW symmetry, we need to look at EW gauge boson processes that are longitudinally dominated at high energy. To determine the channels to study, we first calculate diboson helicity amplitudes in the high energy limit [74,76-78]. The fully longitudinal double EW gauge boson production modes are

$$
\begin{aligned}
\mathcal{A}\left(q_{+} \bar{q}_{-} \rightarrow W_{L}^{+} W_{L}^{-}\right)= & -i \frac{e^{2} Q_{q}}{2 c_{W}^{2}} \sin \theta+\mathcal{O}\left(\hat{s}^{-1}\right), \\
\mathcal{A}\left(q_{-} \bar{q}_{+} \rightarrow W_{L}^{+} W_{L}^{-}\right)= & i \frac{e^{2} T_{3}^{q}}{6 c_{W}^{2} s_{W}^{2}}\left(3 c_{W}^{2}+2 T_{3}^{q} s_{W}^{2}\right) \sin \theta \\
& +\mathcal{O}\left(\hat{s}^{-1}\right), \\
\mathcal{A}\left(q_{-} \bar{q}_{+}^{\prime} \rightarrow W_{L}^{ \pm} Z_{L}\right)= & -i \frac{e^{2} T_{3}^{q}}{\sqrt{2} s_{W}^{2}} \sin \theta+\mathcal{O}\left(\hat{s}^{-1}\right), \\
\mathcal{A}\left(q_{ \pm} \bar{q}_{\mp}^{\prime} \rightarrow Z_{L} Z_{L}\right)= & \mathcal{O}\left(\hat{s}^{-1}\right),
\end{aligned}
$$


where $\sqrt{\hat{S}}$ is the partonic center of mass energy, the subscript $L$ on EW gauge bosons indicates longitudinal polarization, and the subscripts on the quarks indicate quark helicity. For $W^{+} W^{-}$production $\theta$ is the angle between $W^{+}$and initial state quark, and for $W Z$ production $\theta$ is the angle between the $W$ and the initial state quark. $Q_{q}$ is the quark $q$ 's charge, $T_{3}^{q}$ is the quark $q$ 's isospin, and $c_{W}=\cos \theta_{W}, s_{W}=\sin \theta_{W}$ is the weak mixing angle.

As expected, the fully longitudinal EW gauge boson pair production modes $W^{+} W^{-}$and $W Z$ persist at high energy. However, so do transversely polarized gauge bosons with opposite helicities:

$$
\begin{aligned}
\mathcal{A}\left(q_{-} \bar{q}_{+} \rightarrow W_{ \pm}^{+} W_{\mp}^{-}\right)= & \mp i \frac{e^{2}}{2 s_{W}^{2}} \frac{1+2 T_{3}^{q} \cos \theta}{1 \pm \cos \theta} \sin \theta+\mathcal{O}\left(\hat{s}^{-1}\right), \\
\mathcal{A}\left(q_{-} \bar{q}_{+}^{\prime} \rightarrow W_{ \pm}^{ \pm} Z_{\mp}\right)= & \mp i \frac{e^{2}}{\sqrt{2} s_{W}^{2} c_{W}}\left(g_{L}^{q^{\prime} Z}(1+\cos \theta)\right. \\
& \left.+g_{L}^{q Z}(1-\cos \theta)\right) \frac{\sin \theta}{1 \pm \cos \theta}+\mathcal{O}\left(\hat{s}^{-1}\right), \\
\mathcal{A}\left(q_{-} \bar{q}_{+} \rightarrow Z_{+} Z_{-}\right)= & 2 i \frac{e^{2}}{s_{W}^{2} c_{W}^{2}} g_{L}^{q Z^{2}} \sqrt{\frac{1-\cos \theta}{1+\cos \theta}}+\mathcal{O}\left(\hat{s}^{-1}\right), \\
\mathcal{A}\left(q_{+} \bar{q}_{-} \rightarrow Z_{+} Z_{-}\right)= & -2 i \frac{e^{2}}{s_{W}^{2} c_{W}^{2}} g_{R}^{q Z^{2}} \sqrt{\frac{1+\cos \theta}{1-\cos \theta}}+\mathcal{O}\left(\hat{s}^{-1}\right),
\end{aligned}
$$

where the subscript \pm on EW gauge bosons indicate the transverse helicities, for $Z Z$ final state $\theta$ is the angle between the initial state quark and $Z_{+}$, and

$$
g_{L}^{q Z}=T_{3}^{q}-Q_{q} s_{W}^{2}, \quad \text { and } \quad g_{R}^{q Z}=-Q_{q} s_{W}^{2}
$$

All other amplitudes are either zero or suppressed at high energies:

$$
\begin{aligned}
\mathcal{A}\left(q_{ \pm} \bar{q}_{\mp} \rightarrow W_{ \pm}^{ \pm} W_{L}^{\mp}\right) & \sim \mathcal{A}\left(q_{-} \bar{q}_{+}^{\prime} \rightarrow W_{ \pm}^{ \pm} Z_{L}\right) \\
& \sim \mathcal{A}\left(q_{-} \bar{q}_{+}^{\prime} \rightarrow Z_{ \pm} W_{L}^{ \pm}\right) \\
& \sim \mathcal{A}\left(q_{ \pm} \bar{q}_{\mp} \rightarrow Z_{ \pm} Z_{L}\right) \sim \mathcal{O}\left(\hat{s}^{-1 / 2}\right), \\
\mathcal{A}\left(q_{ \pm} \bar{q}_{\mp} \rightarrow W_{ \pm}^{+} W_{ \pm}^{-}\right) & \sim \mathcal{A}\left(q_{-} \bar{q}_{+}^{\prime} \rightarrow W_{ \pm}^{ \pm} Z_{ \pm}\right) \\
& \sim \mathcal{A}\left(q_{ \pm} \bar{q}_{\mp} \rightarrow Z_{ \pm} Z_{ \pm}\right) \sim \mathcal{O}\left(\hat{s}^{-1}\right), \\
\mathcal{A}\left(q_{+} \bar{q}_{-} \rightarrow W_{ \pm}^{+} W_{\mp}^{-}\right) & =\mathcal{A}\left(q_{+} \bar{q}_{-}^{\prime} \rightarrow W_{\lambda}^{ \pm} Z_{\lambda^{\prime}}\right)=0 .
\end{aligned}
$$

From Eqs. (3) and (4) it is clear that double EW gauge boson production is not longitudinally dominated. Indeed, even though both fully longitudinal and transverse polarizations persist at high energy, as shown in Fig. 1(a,b) $W W$ and $W Z$ production are strongly dominated by the transverse polarizations. Here we use CTEQ6L1 parton distribution functions (pdfs) [79] implemented in LHAPDF [80] via ManeParse [81]. This is particularly striking in $W W$ production where at high energies $90 \%-95 \%$ of the $W \mathrm{~s}$ are transversely polarized, while $W Z$ production is $60 \%-70 \%$ transversely polarized. Hence, to find the longitudinally polarized signal and observe EW restoration in $q \bar{q}^{\prime} \rightarrow V V^{\prime}$, either the differences in the angular distributions of the gauge bosons must be exploited or their polarizations must be tagged, which is very difficult [12,75,77,82-87]. There is also an additional complication that the gauge bosons are not final state particles and different gauge boson polarizations interfere with each other [75,78,82-84].

These complications do not arise in EW gauge boson production in association with a Higgs [19]:

$$
\begin{aligned}
& \mathcal{A}\left(q_{+} \bar{q}_{-} \rightarrow Z_{L} h\right)= \pm i \frac{e^{2} g_{R}^{q Z}}{2 c_{W}^{2} s_{W}^{2}} \sin \theta+\mathcal{O}\left(\hat{s}^{-1}\right), \\
& \mathcal{A}\left(q_{-} \bar{q}_{+} \rightarrow Z_{L} h\right)= \pm i \frac{e^{2} g_{L}^{q Z}}{2 c_{W}^{2} s_{W}^{2}} \sin \theta+\mathcal{O}\left(\hat{s}^{-1}\right), \\
& \mathcal{A}\left(q_{-} \bar{q}_{+}^{\prime} \rightarrow W_{L}^{ \pm} h\right)=-i \frac{e^{2}}{2 \sqrt{2} s_{W}^{2}} \sin \theta+\mathcal{O}\left(\hat{s}^{-1}\right), \\
& \mathcal{A}\left(q_{ \pm} \bar{q}_{\mp} \rightarrow Z_{ \pm} h\right) \sim \mathcal{A}\left(q_{-} \bar{q}_{+}^{\prime} \rightarrow W_{L}^{ \pm} h\right) \sim \mathcal{O}\left(\hat{s}^{-1 / 2}\right), \\
& \mathcal{A}\left(q_{+} \bar{q}_{-}^{\prime} \rightarrow W_{ \pm}^{ \pm} h\right)=\mathcal{A}\left(q_{+} \bar{q}_{-}^{\prime} \rightarrow W_{\mp}^{ \pm} h\right)=0 .
\end{aligned}
$$

The longitudinal polarizations persist at high energy while transverse polarizations decrease with energy. This is even more clear in Figs. 1(c,d), where the transverse polarizations make sub-percent level contributions to the total rate at high transverse momentum. Since $q \bar{q}^{\prime} \rightarrow V h$ is quickly dominated by longitudinally polarized gauge bosons, there is no need to use polarization tagging to get a longitudinally enriched signal. Hence, this channel is a prime candidate to observe EW restoration and the focus of our phenomenological analysis.

\section{B. EW restoration}

To observe EW restoration, the $S U(2) \times U(1)$ symmetric phase with $v=0$ should be considered. In this phase, the EW gauge bosons and SM fermions are massless. To obtain $v=0$, the $\mu^{2}$ parameter in Eq. (1) must be zero or negative. Hence, in principle the Higgs field could have a nonzero mass. We will enforce the tree level relationships between the Higgs mass $m_{h}$, the $\mu^{2}$ parameter, and the vev then take the limit $v \rightarrow 0$ :

$$
\begin{aligned}
& \mu^{2}=\lambda v^{2} \underset{v \rightarrow 0}{\rightarrow} 0, \\
& m_{h}^{2}=2 \lambda v^{2} \underset{v \rightarrow 0}{\rightarrow} 0 .
\end{aligned}
$$




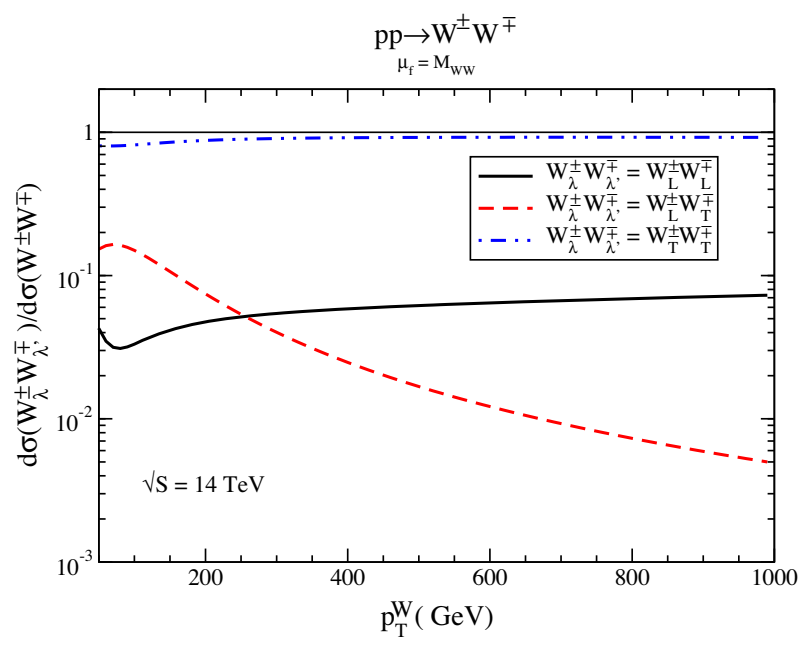

(a)

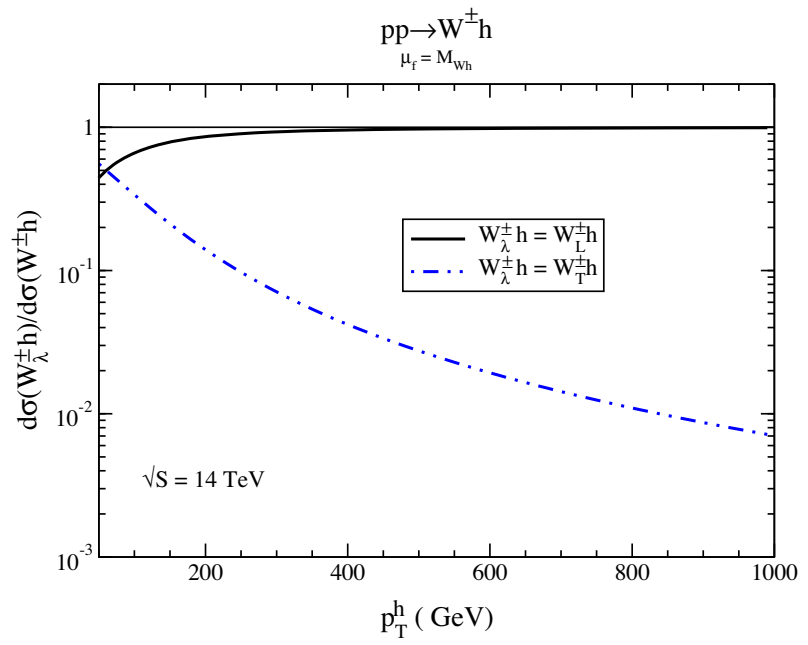

(c)

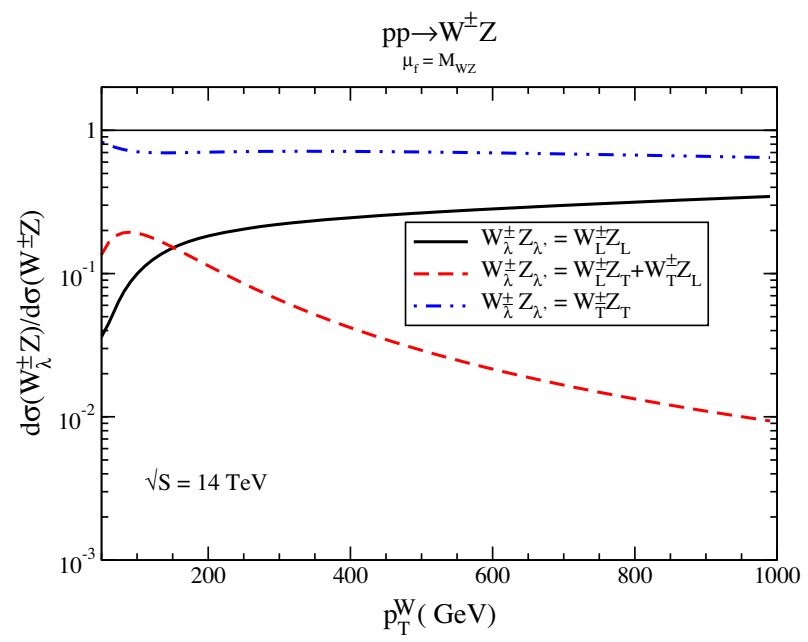

(b)

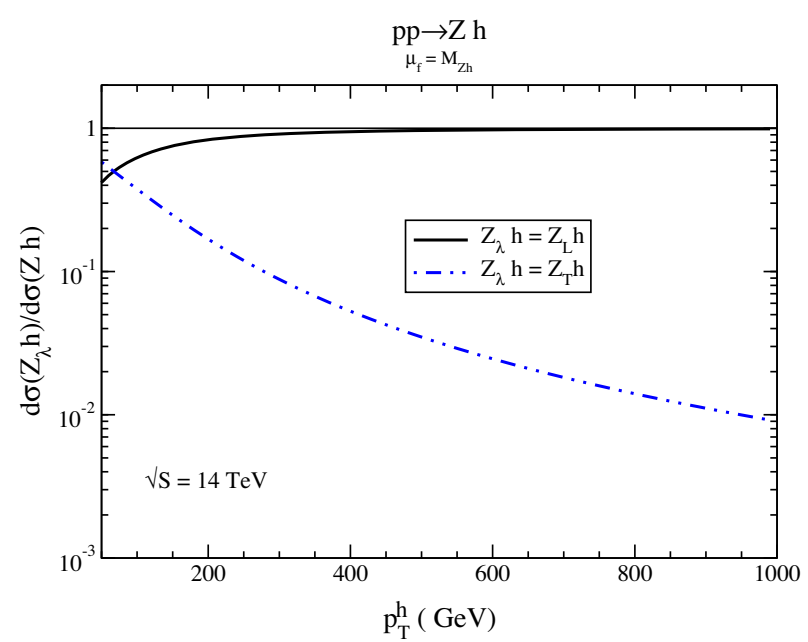

(d)

FIG. 1. Ratio of transverse momentum distributions of polarized gauge boson production to the total distribution summed over polarizations. These are shown using $W$ transverse momentum, $p_{T}^{W}$, for (a) $W^{ \pm} W^{\mp}$ and (b) $W^{ \pm} Z$; and Higgs transverse momentum, $p_{T}^{h}$, for (c) $W^{ \pm} h$ and (d) $Z h$. The gauge boson polarizations are (blue dash-dot-dot) fully longitudinal, (black solid) fully transverse, and (red dashed) longitudinal + transverse. The lab frame energy is the HL-LHC energy of $\sqrt{S}=14 \mathrm{TeV}$. The subscript $T$ on the gauge bosons indicate summed over transverse polarizations.

That is, we consider a massless Higgs doublet field consistent with the parameter relationships in the SM.

Once the $S U(2) \times U(1)$ symmetry is restored, calculations should be performed in the unbroken phase. The relevant degrees of freedom are the $S U(2)$ gauge boson multiplet, the hypercharge gauge boson, the Higgs doublet, the left-handed fermion doublets, and the right-handed fermion singlets. All fields are massless. However, when considering collider phenomenology a couple complications arise. First, vector bosons, Goldstones, and the Higgs boson are not final state particles. Hence, their charges can be distinguished by the detector via their decay products. This separates the components of the Higgs doublet and the $S U(2) \times U(1)$ gauge boson multiplets. Second, each flavor of quark has a different pdf and the pdfs distinguish the components of the quark doublets. Each of these effects break EW symmetry at the detector level.

With those considerations we compute Goldstone boson and Higgs production helicity amplitudes with initial and final states considered component-by-component. For intermediate particles the massless gauge bosons of the unbroken $S U(2) \times U(1)$ are used. The relevant helicity amplitudes for diboson production are 


$$
\begin{aligned}
\mathcal{A}\left(q_{+} \bar{q}_{-} \rightarrow G^{0} h\right) & =-\frac{e^{2} g_{R}^{q Z}}{2 c_{W}^{2} s_{W}^{2}} \sin \theta, \\
\mathcal{A}\left(q_{-} \bar{q}_{+} \rightarrow G^{0} h\right) & =\frac{e^{2} g_{L}^{q Z}}{2 c_{W}^{2} s_{W}^{2}} \sin \theta, \\
\mathcal{A}\left(q_{-} \bar{q}_{+} \rightarrow G^{ \pm} h\right) & =\mp i \frac{e^{2}}{2 \sqrt{2} s_{W}^{2}} \sin \theta, \\
\mathcal{A}\left(q_{-} \bar{q}_{+} \rightarrow G^{ \pm} G^{0}\right) & =\frac{e^{2}}{2 \sqrt{2} s_{W}^{2}} \sin \theta, \\
\mathcal{A}\left(q_{+} \bar{q}_{-} \rightarrow G^{+} G^{-}\right) & =-i \frac{e^{2} Q_{q}}{2 c_{W}^{2}} \sin \theta, \\
\mathcal{A}\left(q_{-} \bar{q}_{+} \rightarrow G^{+} G^{-}\right) & =-i \frac{e^{2} T_{3}^{q}}{6 c_{W}^{2} s_{W}^{2}}\left(3 c_{W}^{2}+2 T_{3}^{q} s_{W}^{2}\right) \sin \theta .
\end{aligned}
$$

As expected from the Goldstone boson equivalence theorem, the Goldstone boson production amplitudes agree with high energy longitudinal gauge boson amplitudes in Eqs. (3) and (7).

To observe how quickly the Goldstone boson equivalence theorem converges in $V h$ production, we define signal strengths as ratios of Higgs transverse momentum, $p_{T}^{h}$, distributions:

$$
\begin{gathered}
\mu_{W h}=\frac{d \sigma\left(p p \rightarrow W^{ \pm} h\right) / d p_{T}^{h}}{d \sigma\left(p p \rightarrow G^{ \pm} h\right) / d p_{T}^{h}}, \\
\mu_{Z h}=\frac{d \sigma(p p \rightarrow Z h) / d p_{T}^{h}}{d \sigma\left(p p \rightarrow G^{0} h\right) / d p_{T}^{h}} .
\end{gathered}
$$

While $\sqrt{\hat{s}}$ is the relevant quantity for the convergence of the GBET, we use $p_{T}^{h}$ since it is more easily reconstructable when there is missing energy from gauge boson decays. The signal strengths are shown in Fig. 2 for both the HL-LHC with lab frame energy $\sqrt{S}=14 \mathrm{TeV}$ and the HE-LHC with $\sqrt{S}=27 \mathrm{TeV}$. While there is a very large difference between the $V h$ and Goldstone boson plus Higgs distributions at low transverse momentum, they converge fairly quickly. At transverse moment of $p_{T}^{h} \sim 400 \mathrm{GeV}$, the $V h$ and $G h$ distributions agree at the $\sim 80 \%$ level.

Both $\mu_{W h}$ and $\mu_{Z h}$ are in good agreement for the entire $p_{T}^{h}$ range at the HL- and HE-LHC. Hence, a uniform signal strength can be defined for both $W^{ \pm} h$ and $Z h$ :

$$
\mu_{V h}=\mu_{W h}=\mu_{Z h} .
$$

Then both $W^{ \pm} h$ and $Z h$ distributions can be fit to the same parameter, making the combination of these measurements straightforward.

\section{SIGNAL STRENGTH AND LIKELIHOOD}

We now turn to how to observe EW restoration in the EW gauge boson plus Higgs boson production via the

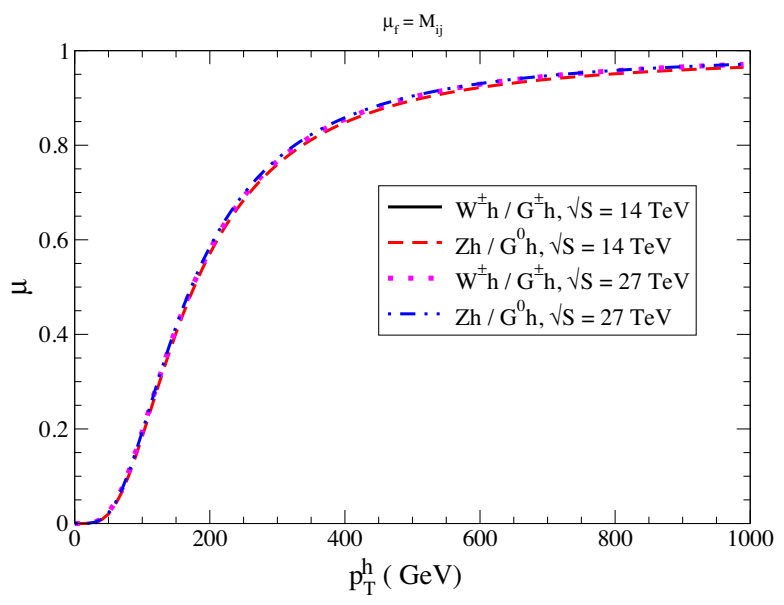

FIG. 2. Signal strengths (black solid) $\mu_{W h}$, (red dashed) $\mu_{Z h}$ at $\sqrt{S}=14 \mathrm{TeV}$ and (magenta dotted) $\mu_{W h}$, (blue dash-dot-dot) $\mu_{Z h}$ at $\sqrt{S}=27 \mathrm{TeV}$.

signal strength in Eq. (11). As discussed before, one immediate issue is that the vector and scalar bosons are not final state particles, and their decay products are detected. The complication is that although the production of $V h$ occurs at high energies, the decays of the vector boson and Higgs occur at the EW scale $\sim 100 \mathrm{GeV}$ where the Goldstone boson equivalence theorem is not a good approximation.

To extract the signal strength [Eq. (11)], the detector level events need to be unfolded [88-90] to the partonic $q \bar{q} \rightarrow V h$ level. There are many modern machine learning [91-94] methods to unfold events. However, we are primarily interested in only the Higgs transverse momentum distribution. Hence, we adapt the unfolding method in Ref. [95] and use a simple one-dimensional likelihood function method. For each bin of $p_{T}^{h}$ we define a likelihood function:

$$
\begin{aligned}
\mathcal{L}_{i}\left(\Delta \sigma_{1}^{V h}, \Delta \sigma_{2}^{V h}, \ldots\right) \\
=\frac{\left(\sum_{j} \Delta \sigma_{j}^{V h} \varepsilon_{i j} L+B_{i}\right)^{n_{o b s, i}}}{n_{o b s, i} !} e^{-\sum_{j} \Delta \sigma_{j}^{V h} \varepsilon_{i j} L-B_{i}},
\end{aligned}
$$

where $i$ labels each $p_{T}^{h}$ bin; $B_{i}$ is the expected number of background events and $n_{o b s, i}$ the total number of observed events in the $i$ th bin; $L$ is the integrated luminosity; $\Delta \sigma_{i}^{V h}$ is the partonic cross section in each bin; and $\epsilon_{i j}$ is an efficiency matrix. The efficiency matrix takes into account detector effects, branching ratios, parton showering, and hadronization. Using the uniform signal strength in Eq. (11), the binned likelihood function is

$$
\begin{aligned}
& \mathcal{L}_{i}\left(\mu_{V h}^{1}, \mu_{V h}^{2}, \ldots\right) \\
& =\frac{\left(\sum_{j} \mu_{V h}^{j} \Delta \sigma_{j}^{G h} \varepsilon_{i j} L+B_{i}\right)^{n_{o b s, i}}}{n_{o b s, i} !} e^{-\sum_{j} \mu_{V h}^{j} \Delta \sigma_{j}^{G h} \varepsilon_{i j} L-B_{i}},
\end{aligned}
$$


where $\mu_{V h}^{j}$ is the signal strength and $\Delta \sigma_{j}^{G h}$ the Goldstone boson plus Higgs production rate in the $j$ th Higgs transverse momentum bin. For $Z h$ production the relevant Goldstone process is $G^{0} h$ and for $W^{ \pm} h$ it is $G^{ \pm} h$.

The efficiency matrix takes care of the probability that a parton level event in the $i$ th bin is in the $j$ th bin at the detector level. To calculate $\epsilon_{i j}$ we generate $V h$ events in MADGRAPH5_aMC@NLO [96] with parton showers and hadronization via PYTHIA8 [97] and detector effects via DELPHES3 [98]. By comparing detector level reconstructed $p_{T}^{h}$ to the parton level information from MADGRAPH5_aMC@NLO, the efficiency matrix $\epsilon_{i j}$ can be determined.

Finally, we use the global likelihood across all bins

$$
\mathcal{L}\left(\mu_{V h}^{1}, \mu_{V h}^{2}, \ldots\right)=\prod_{i} \mathcal{L}_{i}\left(\mu_{V h}^{1}, \mu_{V h}^{2}, \ldots\right) \operatorname{Pois}\left(n_{o b s, i} \mid S_{i}+B_{i}\right),
$$

where $\operatorname{Pois}(x \mid y)$ is a conditional Poisson distribution and $S_{i}$ is the expected number of signal events in the $i$ th bin. Now, given a number of observed events $n_{o b s, i}$, Eq. (14) is maximized to determine the binned signal strengths $\mu_{V h}^{i}$.

\section{COLLIDER ANALYSIS}

We now turn to extracting our signal from background. To get larger rates and clean signals, we consider $h \rightarrow b b$ and leptonic decays of the EW gauge boson. Signal events are decomposed into six categories:

(1) Two lepton final states, $Z h \rightarrow \ell^{+} \ell^{-} b \bar{b}$, with either

(a) exactly two jets from the Higgs or

(b) three or more jets.

(2) One lepton final states, $W h \rightarrow \ell \nu b \bar{b}$, with either

(a) exactly two jets from the Higgs or

(b) exactly three jets.

(3) Zero lepton final states, $Z h \rightarrow \nu \nu b \bar{b}$, with either

(a) exactly two jets from $h \rightarrow b b$ or

(b) exactly three jets.

Note, for each signal with different multiplicities of jets, the efficiency matrix $\epsilon_{i j}$ in Eq. (13) must be recalculated to map onto the partonic $q \bar{q} \rightarrow V h$ event.

The major backgrounds are: QCD production of $V+l l$, $V+\mathrm{HF}, V+c l$ as well as top pair, single top and vector boson pair. Here $l=u, d, s, g$, and HF indicates "heavy flavor": $b b, b c, c c, b l$. For the zero and one-lepton signals, we include backgrounds from missing leptons. The missing lepton rate is estimated by using the default setting of DELPHES3.

\section{A. Simulation}

We use MADGRAPH5_aMC@NLO [96] for parton level generation and heavy particle decay, PYTHIA8 [97] for parton showers and hadronization, DELPHES3 [98] for fast detector simulation. Finally, MLM jet matching [99] up to one additional jet is used for background simulation.

In the detector simulation, we use the default CMS card with some modifications. While there is a DELPHES3 card for Future Circular Colliders (FCC) that can be used for $27 \mathrm{TeV}$, for simplicity and direct comparison with $14 \mathrm{TeV}$ results we use the CMS card for both the HL and HE-LHC. The FCC DELPHES 3 card has electron tracking efficiency of 99\% for transverse momentum greater than $1 \mathrm{GeV}$. Hence, we use the same basic acceptance cuts for leptons at both the $14 \mathrm{TeV}$ HL-LHC and $27 \mathrm{TeV}$ HE-LHC [100]:

(i) Lepton transverse momentum, $p_{T}^{\ell}$ :

$$
p_{T}^{\ell} \geq 27 \mathrm{GeV} .
$$

(ii) Lepton, $\eta_{\ell}$, and jet, $\eta_{j}$, rapidity:

$$
\left|\eta_{\ell}\right| \leq 2.5, \quad\left|\eta_{j}\right| \leq 5.0
$$

(iii) Minimum separation between jets, $j$, and leptons, $\ell$ :

$$
\Delta R_{j j} \geq 0.4, \quad \Delta R_{j \ell} \geq 0.4, \quad \Delta R_{\ell \ell} \geq 0.4 .
$$

(iv) Electron isolation: PTRatioMax $=0.43^{2}$ considering particles with $p_{T}>0.5 \mathrm{GeV}$ and within a cone of radius $\Delta R<0.3$.

(v) Muon isolation: PTRatioMax $=0.25$ considering particles with $p_{T}>0.5 \mathrm{GeV}$ and within a cone of radius $\Delta R<0.4$.

The minimum jet transverse momentum, $p_{T}^{j}$, requirement is different between 14 and $27 \mathrm{TeV}$ :

(i) At $14 \mathrm{TeV}$ :

$$
p_{T}^{j} \geq 20 \mathrm{GeV} .
$$

(ii) At $27 \mathrm{TeV}[101]$ :

$$
p_{T}^{j} \geq 30 \mathrm{GeV} .
$$

Finally, since our signal is rich in $b$-quarks, we also use a $b$ tagging rate of 0.70 with mis-tag rates of 0.125 for charm jets and 0.003 for light jets [102].

\section{B. Classification}

To classify signal from background, we use "precuts" followed by a DNN. The precuts are basic multiplicity and invariant mass cuts to help separate signal and background:

(i) For the two lepton signals $\left(n_{\ell}=2\right)$ we require exactly two same flavor, opposite sign leptons that reconstruct the $Z$ mass $\left|m_{\ell \ell}-m_{Z}\right| \leq 10 \mathrm{GeV}$, where $m_{\ell \ell}$ is the dilepton invariant mass. In

\footnotetext{
${ }^{2}$ See Eq. (3.1) of Ref. [98] for definition of PTRatioMax.
} 
addition, we require at least two jets $\left(n_{j} \geq 2\right)$ passing the cuts in Eqs. (16), (18), and (19).

(ii) For both the zero $\left(n_{\ell}=0\right)$ and one lepton $\left(n_{\ell}=1\right)$ signal we require either two or three jets $\left(n_{j}=2,3\right)$ to pass the cuts in Eqs. (16), (18), and (19).

For all signals we require exactly two $b$-tagged jets $\left(n_{b}=2\right)$.

After events pass the pre-cuts, a DNN is used to further classify signal and background. The inputs of the DNN are high-level reconstructed variables and are detailed in Appendix. The DNN is a binary classifier consisting of three hidden layers with $2^{10}, 2^{12}$, and $2^{10}$ nodes. We adopt LeakyReLU [103] for non-linearity, use batch normalization between layers, and the output layer uses softmax to create a probability. We use cross entropy as the loss function with an $L 2$ penalty:

$$
L=-y_{s} \log p-\left(1-y_{s}\right) \log (1-p)+\lambda\|W\|^{2},
$$

where $y_{s}$ is the signal indicator with $y_{s}=1$ for signal and $y_{s}=0$ for background, $p$ is the predicted signal probability, and $\|W\|^{2}$ is the matrix norm of the weight matrices. While the same DNN structure is used for all six categories, the $L 2$ penalty value $\lambda$ changes.

Cut flow tables and signal significances are given in Table I for two lepton categories, Table II for the one lepton categories, and Table III for the zero lepton category at both 14 and $27 \mathrm{TeV}$. The significances are calculated for the benchmark luminosities of $3 \mathrm{ab}^{-1}$ for the HL-LHC and $15 \mathrm{ab}^{-1}$ for the HE-LHC. We use the asymptotic formula for a discovery significance with Poisson statistics

$$
\sigma=\sqrt{2\left(\left(N_{s}+N_{b}\right) \times \log \left(1+\frac{N_{s}}{N_{b}}\right)-N_{s}\right)},
$$

TABLE I. Cut flow table and signal significance after the DNN for the two lepton categories. The significances correspond to $3 \mathrm{ab}^{-1}$ at

\begin{tabular}{|c|c|c|c|c|c|c|c|c|}
\hline & \multicolumn{4}{|c|}{$14 \mathrm{TeV}$} & \multicolumn{4}{|c|}{$27 \mathrm{TeV}$} \\
\hline & \multicolumn{2}{|c|}{$n_{j}=2$} & \multicolumn{2}{|c|}{$n_{j}=3$} & \multicolumn{2}{|c|}{$n_{j}=2$} & \multicolumn{2}{|c|}{$n_{j}=3$} \\
\hline & Pre-Cut & DNN & Pre-Cut & DNN & Pre-Cut & DNN & Pre-Cut & DNN \\
\hline$h_{b b} Z_{\ell \ell}$ & $1.1 \mathrm{fb}$ & $0.22 \mathrm{fb}$ & $1.1 \mathrm{fb}$ & $0.23 \mathrm{fb}$ & $2.0 \mathrm{fb}$ & $0.87 \mathrm{fb}$ & $1.6 \mathrm{fb}$ & $1.2 \mathrm{fb}$ \\
\hline$Z+\mathrm{HF}$ & $300 \mathrm{fb}$ & $1.4 \mathrm{fb}$ & $530 \mathrm{fb}$ & $3.3 \mathrm{fb}$ & $580 \mathrm{fb}$ & $16 \mathrm{fb}$ & $780 \mathrm{fb}$ & $120 \mathrm{fb}$ \\
\hline$t t$ & $27 \mathrm{fb}$ & $0.14 \mathrm{fb}$ & $69 \mathrm{fb}$ & $0.095 \mathrm{fb}$ & $92 \mathrm{fb}$ & $1.6 \mathrm{fb}$ & $180 \mathrm{fb}$ & $19 \mathrm{fb}$ \\
\hline Single top & $0.85 \mathrm{fb}$ & $0.0036 \mathrm{fb}$ & $3.5 \mathrm{fb}$ & $0.0041 \mathrm{fb}$ & $2.9 \mathrm{fb}$ & $0.047 \mathrm{fb}$ & $11 \mathrm{fb}$ & $1.0 \mathrm{fb}$ \\
\hline $\mathrm{Zcl}$ & $0.18 \mathrm{fb}$ & $0.0036 \mathrm{fb}$ & $2.1 \mathrm{fb}$ & $0.025 \mathrm{fb}$ & $0.75 \mathrm{fb}$ & $0.034 \mathrm{fb}$ & $6.4 \mathrm{fb}$ & $0.94 \mathrm{fb}$ \\
\hline Zll & $0.68 \mathrm{fb}$ & $0.019 \mathrm{fb}$ & $13 \mathrm{fb}$ & $0.20 \mathrm{fb}$ & $2.0 \mathrm{fb}$ & $0.096 \mathrm{fb}$ & $27 \mathrm{fb}$ & $4.1 \mathrm{fb}$ \\
\hline$V V^{\prime}$ & $4.8 \mathrm{fb}$ & $0.026 \mathrm{fb}$ & $5.4 \mathrm{fb}$ & $0.051 \mathrm{fb}$ & $6.5 \mathrm{fb}$ & $0.22 \mathrm{fb}$ & $7.8 \mathrm{fb}$ & $1.5 \mathrm{fb}$ \\
\hline Signal significance & & 9.4 & & 6.5 & & 25 & & 13 \\
\hline
\end{tabular}
$14 \mathrm{TeV}$ and $15 \mathrm{ab}^{-1}$ at $27 \mathrm{TeV}$. All backgrounds include possible decays leading to events with and without missing leptons.

TABLE II. Cut flow table and signal significance after the DNN for the one lepton categories. The significances correspond to 3 ab ${ }^{-1}$

\begin{tabular}{|c|c|c|c|c|c|c|c|c|}
\hline & \multicolumn{4}{|c|}{$14 \mathrm{TeV}$} & \multicolumn{4}{|c|}{$27 \mathrm{TeV}$} \\
\hline & \multicolumn{2}{|c|}{$n_{j}=2$} & \multicolumn{2}{|c|}{$n_{j}=3$} & \multicolumn{2}{|c|}{$n_{j}=2$} & \multicolumn{2}{|c|}{$n_{j}=3$} \\
\hline & Pre-Cut & DNN & Pre-Cut & DNN & Pre-Cut & DNN & Pre-Cut & DNN \\
\hline$h_{b b} W_{\ell \nu}$ & $12 \mathrm{fb}$ & $6.1 \mathrm{fb}$ & $7.3 \mathrm{fb}$ & $0.38 \mathrm{fb}$ & $19 \mathrm{fb}$ & $9.6 \mathrm{fb}$ & $9.8 \mathrm{fb}$ & $1.2 \mathrm{fb}$ \\
\hline$W+\mathrm{HF}$ & $580 \mathrm{fb}$ & $38 \mathrm{fb}$ & $640 \mathrm{fb}$ & $0.035 \mathrm{fb}$ & $790 \mathrm{fb}$ & $43 \mathrm{fb}$ & $940 \mathrm{fb}$ & $0.33 \mathrm{fb}$ \\
\hline$Z+\mathrm{HF}$ & $310 \mathrm{fb}$ & $8.5 \mathrm{fb}$ & $380 \mathrm{fb}$ & $9.7 \times 10^{-5} \mathrm{fb}$ & $640 \mathrm{fb}$ & $21 \mathrm{fb}$ & $670 \mathrm{fb}$ & $0.048 \mathrm{fb}$ \\
\hline$t t$ & $150 \mathrm{fb}$ & $15 \mathrm{fb}$ & $560 \mathrm{fb}$ & $0.30 \mathrm{fb}$ & $580 \mathrm{fb}$ & $28 \mathrm{fb}$ & $1500 \mathrm{fb}$ & $0.93 \mathrm{fb}$ \\
\hline Single top & $11 \mathrm{fb}$ & $1.1 \mathrm{fb}$ & $68 \mathrm{fb}$ & $0.053 \mathrm{fb}$ & $36 \mathrm{fb}$ & $1.7 \mathrm{fb}$ & $100 \mathrm{fb}$ & $0.12 \mathrm{fb}$ \\
\hline Wcl & $4.9 \mathrm{fb}$ & $0.46 \mathrm{fb}$ & $12 \mathrm{fb}$ & $2.5 \times 10^{-3} \mathrm{fb}$ & $8.0 \mathrm{fb}$ & $0.56 \mathrm{fb}$ & $19 \mathrm{fb}$ & $0.027 \mathrm{fb}$ \\
\hline Wll & $10 \mathrm{fb}$ & $1.2 \mathrm{fb}$ & $36 \mathrm{fb}$ & $0.021 \mathrm{fb}$ & $28 \mathrm{fb}$ & $2.7 \mathrm{fb}$ & $92 \mathrm{fb}$ & $0.34 \mathrm{fb}$ \\
\hline$Z c l$ & $0.15 \mathrm{fb}$ & $4.2 \times 10^{-3} \mathrm{fb}$ & $0.51 \mathrm{fb}$ & $0 \mathrm{fb}$ & $0.62 \mathrm{fb}$ & $0.012 \mathrm{fb}$ & $1.8 \mathrm{fb}$ & $7.2 \times 10^{-5} \mathrm{fb}$ \\
\hline Zll & $0.49 \mathrm{fb}$ & $0.014 \mathrm{fb}$ & $2.0 \mathrm{fb}$ & $4.7 \times 10^{-5} \mathrm{fb}$ & $1.5 \mathrm{fb}$ & $0.032 \mathrm{fb}$ & $5.2 \mathrm{fb}$ & $6.0 \times 10^{-4} \mathrm{fb}$ \\
\hline$V V^{\prime}$ & $34 \mathrm{fb}$ & $2.0 \mathrm{fb}$ & $28 \mathrm{fb}$ & $0.015 \mathrm{fb}$ & $41 \mathrm{fb}$ & $1.9 \mathrm{fb}$ & $33 \mathrm{fb}$ & $0.11 \mathrm{fb}$ \\
\hline Signal significance & & 40 & & 28 & & 120 & & 98 \\
\hline
\end{tabular}
at $14 \mathrm{TeV}$ and $15 \mathrm{ab}^{-1}$ at $27 \mathrm{TeV}$. All backgrounds include possible decays leading to events with and without missing leptons. 
TABLE III. Cut flow table and signal significance after the DNN for the zero lepton categories. The significances correspond to $3 \mathrm{ab}^{-1}$ at $14 \mathrm{TeV}$ and $15 \mathrm{ab}^{-1}$ at $27 \mathrm{TeV}$. All backgrounds include possible decays leading to events with and without missing leptons.

\begin{tabular}{|c|c|c|c|c|c|c|c|c|}
\hline & \multicolumn{4}{|c|}{$14 \mathrm{TeV}$} & \multicolumn{4}{|c|}{$27 \mathrm{TeV}$} \\
\hline & \multicolumn{2}{|c|}{$n_{j}=2$} & \multicolumn{2}{|c|}{$n_{j}=3$} & \multicolumn{2}{|c|}{$n_{j}=2$} & \multicolumn{2}{|c|}{$n_{j}=3$} \\
\hline & Pre-Cut & DNN & Pre-Cut & DNN & Pre-Cut & DNN & Pre-Cut & DNN \\
\hline$h_{b b} Z_{\nu \nu}$ & $9.8 \mathrm{fb}$ & $4.7 \mathrm{fb}$ & $6.3 \mathrm{fb}$ & $1.6 \mathrm{fb}$ & $18 \mathrm{fb}$ & $7.9 \mathrm{fb}$ & $9.6 \mathrm{fb}$ & $1.4 \mathrm{fb}$ \\
\hline$W+\mathrm{HF}$ & $310 \mathrm{fb}$ & $7.6 \mathrm{fb}$ & $440 \mathrm{fb}$ & $0.020 \mathrm{fb}$ & $420 \mathrm{fb}$ & $14 \mathrm{fb}$ & $680 \mathrm{fb}$ & $0.028 \mathrm{fb}$ \\
\hline$Z+\mathrm{HF}$ & $2900 \mathrm{fb}$ & $110 \mathrm{fb}$ & $2900 \mathrm{fb}$ & $0.35 \mathrm{fb}$ & $5700 \mathrm{fb}$ & $260 \mathrm{fb}$ & $5000 \mathrm{fb}$ & $0.72 \mathrm{fb}$ \\
\hline$t t$ & $7.6 \mathrm{fb}$ & $0.16 \mathrm{fb}$ & $170 \mathrm{fb}$ & $0.041 \mathrm{fb}$ & $42 \mathrm{fb}$ & $0.22 \mathrm{fb}$ & $460 \mathrm{fb}$ & $0.020 \mathrm{fb}$ \\
\hline Single top & $1.3 \mathrm{fb}$ & $0.035 \mathrm{fb}$ & $22 \mathrm{fb}$ & $0.0091 \mathrm{fb}$ & $1.5 \mathrm{fb}$ & $0.0057 \mathrm{fb}$ & $19 \mathrm{fb}$ & $0.0019 \mathrm{fb}$ \\
\hline Wcl & $1.1 \mathrm{fb}$ & $0.026 \mathrm{fb}$ & $4.2 \mathrm{fb}$ & $5.3 \times 10^{-4} \mathrm{fb}$ & $2.4 \mathrm{fb}$ & $0.059 \mathrm{fb}$ & $7.4 \mathrm{fb}$ & $0.0010 \mathrm{fb}$ \\
\hline$W l l$ & $3.7 \mathrm{fb}$ & $0.087 \mathrm{fb}$ & $19 \mathrm{fb}$ & $0.014 \mathrm{fb}$ & $13 \mathrm{fb}$ & $0.38 \mathrm{fb}$ & $49 \mathrm{fb}$ & $0.028 \mathrm{fb}$ \\
\hline $\mathrm{Zcl}$ & $1.4 \mathrm{fb}$ & $0.15 \mathrm{fb}$ & $4.7 \mathrm{fb}$ & $0.0065 \mathrm{fb}$ & $3.3 \mathrm{fb}$ & $0.23 \mathrm{fb}$ & $9.0 \mathrm{fb}$ & $0.013 \mathrm{fb}$ \\
\hline$Z l l$ & $6.8 \mathrm{fb}$ & $0.78 \mathrm{fb}$ & $26 \mathrm{fb}$ & $0.12 \mathrm{fb}$ & $22 \mathrm{fb}$ & $1.6 \mathrm{fb}$ & $80 \mathrm{fb}$ & $0.20 \mathrm{fb}$ \\
\hline$V V^{\prime}$ & $68 \mathrm{fb}$ & $3.9 \mathrm{fb}$ & $51 \mathrm{fb}$ & $0.084 \mathrm{fb}$ & $89 \mathrm{fb}$ & $4.7 \mathrm{fb}$ & $65 \mathrm{fb}$ & $0.15 \mathrm{fb}$ \\
\hline Signal significance & & 23 & & 84 & & 58 & & 140 \\
\hline
\end{tabular}

where $N_{s}, N_{b}$ are the number of signal and background events, respectively. It is clear that background and signal are well separated.

In Fig. 3 we show the reconstructed vector boson $p_{T}$ distributions after the DNN selection for all six categories. The background is cumulative, and the signal is overlaid. At high energies the signal and background separation is better. This is precisely where we expect to see EW restoration.

\section{RESULTS}

To fit the signal strengths in Eq. (11) we perform pseudoexperiments to sample the binned $p_{T}^{h}$ distribution. After the collider analysis of the previous section, we have a sample of signal and background events. That sample is used to create a probability density function (PDF) for the signal and background $p_{T}^{h}$ distribution. The total number of events is sampled according to a Gaussian distribution with the mean $\nu=S_{\text {tot }}+B_{\text {tot }}$ and standard deviation $\sigma=\sqrt{\nu}$, where the total number of expected signal and background events are

$$
S_{\mathrm{tot}}=\sum_{i} S_{i}, \quad B_{\mathrm{tot}}=\sum_{i} B_{i},
$$

respectively, and $S_{i}, B_{i}$ are the expected number of signal and background events in the $i$ th bin after the DNN, respectively. The total number of events is then distributed according to the $p_{T}^{h}$ PDF. In practice, instead of the Higgs transverse momentum, we use the dilepton $p_{T}$ for two lepton categories. At tree level, this is equivalent to $p_{T}^{h}$ for the $V h$ signal. For the zero and one lepton categories, we do use the reconstructed Higgs $p_{T}$.

For each of the six categories, we perform these pseudoexperiments at $14 \mathrm{TeV}$ and $27 \mathrm{TeV}$. Then the $p_{T}^{h}$ distribution is repeatedly sampled for each pseudoexperiment. These samples determine the number of observed events $n_{o b s, i}$ for each bin in Eqs. (12), (13), and (14). In Eqs. (12), (13), and (14), $S_{i}$ and $B_{i}$ are the same as used to set the mean and standard deviation for $n_{o b s, i}$ sampling. For each pseudoexperiment, we maximize the likelihood function Eq. (14) to find the best fit value for the signal strength and then determine the $68 \% \mathrm{CL}$ on $\mu_{V h}$. For each category, we average the best fit values and error bars over all pseudoexperiments.

In Fig. 4 we show the signal strength mean value and $68 \% \mathrm{CL}$ for each of the 6 categories at 14 and $27 \mathrm{TeV}$ with $3 \mathrm{ab}^{-1}$ and $15 \mathrm{ab}^{-1}$ of data, respectively. As expected, all categories at a given lab frame energy $\sqrt{S}$ have the same central values for $\mu_{V h}$. Also, the two jet categories have much smaller uncertainties than the three jets, indicating little information is gained from the three jet categories.

Now that we have the individual signal strengths, we can combine them. To do this, we create a "global" likelihood that is the product of the likelihoods for the six signal categories. Then we perform the same procedure above with pseudoexperiments for each category to find the central value and $68 \% \mathrm{CL}$ for $\mu_{V h}$. These results are shown in Fig. 5 for both (a) the HL-LHC and (b) the HE-LHC, with the predicted partonic level signal strength overlaid. As can be seen, the extracted central value is indistinguishable from the prediction. In an optimistic scenario, the systematic uncertainty on $V h$ production is expected to be 5\% [101]. The red uncertainty bands show the statistical uncertainty, and the green bands show statistical and a 5\% systematic uncertainty added in quadrature.

At low $p_{T}^{h}$, the signal strength is significantly far from one and then converges to one at higher energies, as expected. Indeed, in the last overflow bin, we find the central value of the signal strength and $68 \% \mathrm{CL}$ to be 


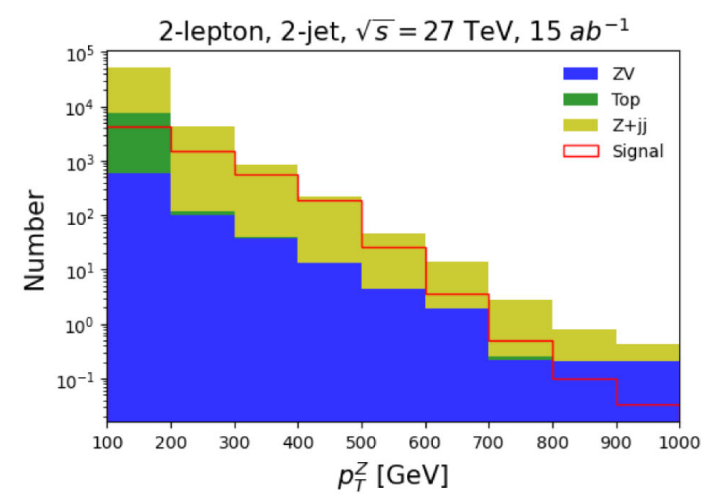

(a)

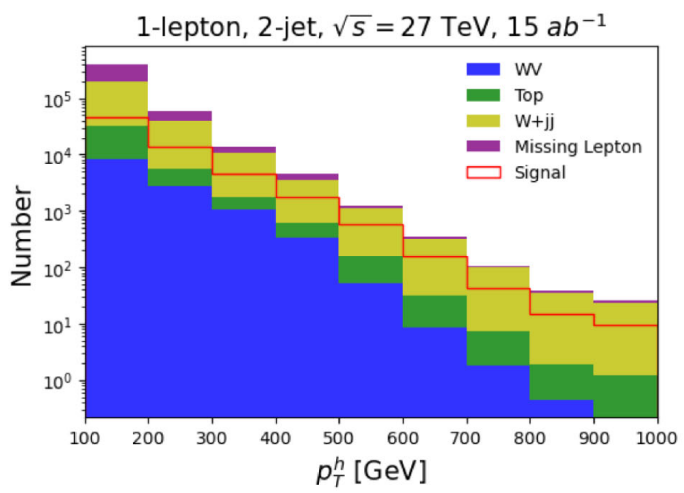

(c)

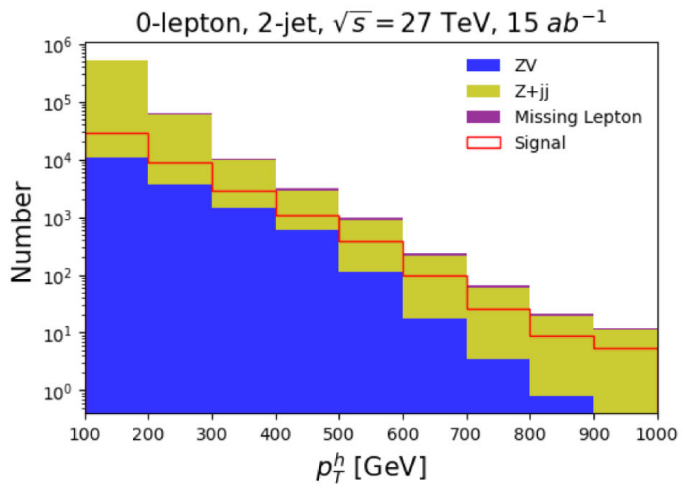

(e)

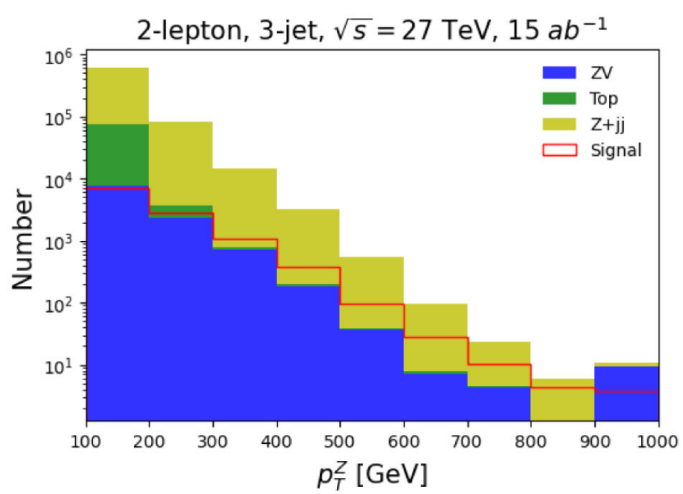

(b)

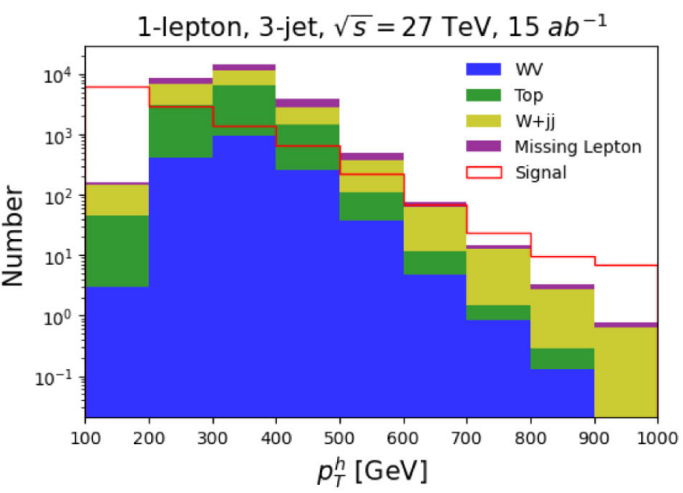

(d)

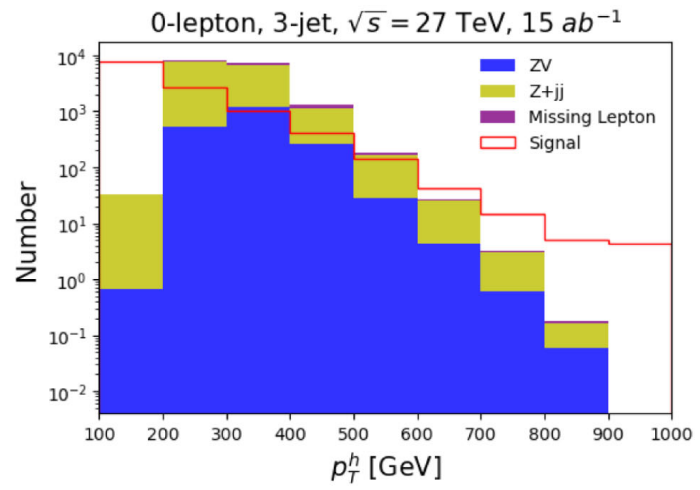

(f)

FIG. 3. The number of events for (red line) signal and (colored bars) background at $27 \mathrm{TeV}$ with $15 \mathrm{ab}^{-1}$ of data. The background is cumulative and the signal overlaid. We show (a,b) two lepton, (c,d) one lepton, and (e,f) zero lepton signal categories for (left-hand side) two jet and (right-hand side) three jet channels. The last bin is an overflow bin. $V+j j$ backgrounds include $V+H F, V+c l$, and $V+l l$. Here, $Z V$ and $W V$ (Top) indicate $V V^{\prime}$ ( $t t$ and single top) backgrounds with no missing leptons. "Missing lepton" indicates backgrounds where a lepton is missed, which is all other backgrounds except those explicitly listed.

$$
\mu_{V h}=\left\{\begin{array}{ll}
1 \pm 0.4 & \text { at the HL-LHC } \\
1 \pm 0.06 & \text { at the HE-LHC }
\end{array} .\right.
$$

That is, the signal agrees with the EW restoration prediction at $40 \%$ at the HL-LHC and $6 \%$ at the HE-LHC. Hence, the $V h$ rate converges to the expected rate with EW symmetry restored. This measurable convergence indicates empirically that the longitudinal modes can be replaced with the Goldstone bosons, and EW restoration can be observed at high energies.

\section{A. Statistical test of EW restoration}

To test how well EW restoration is being observed, one needs to measure how the convergence is improving 


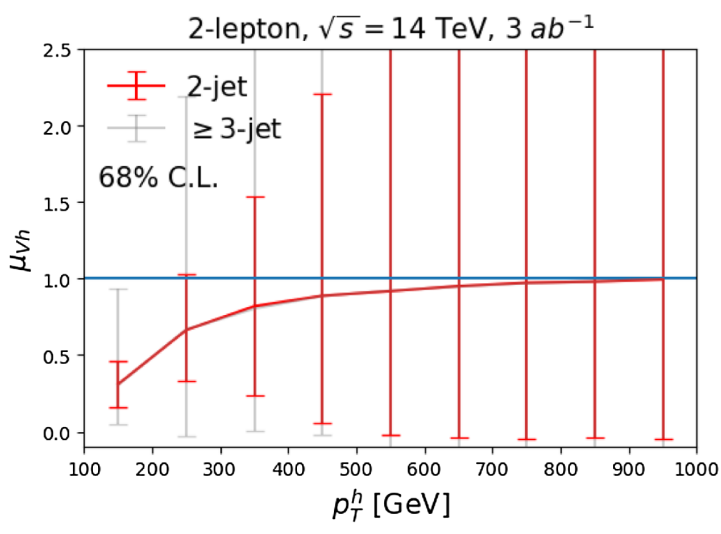

(a)

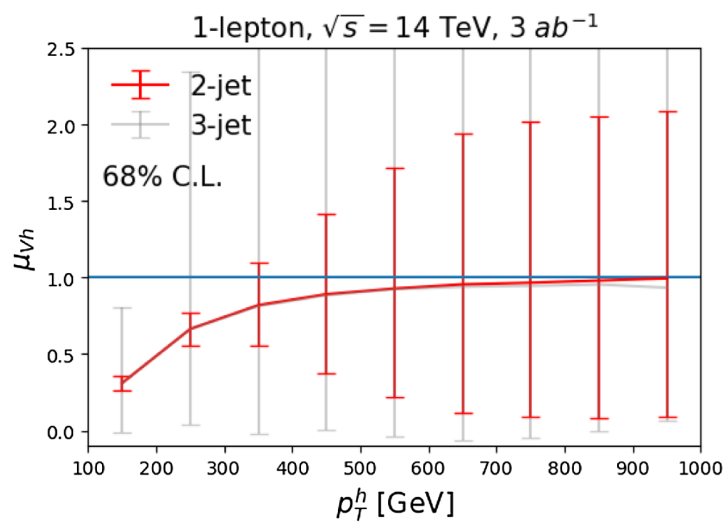

(c)

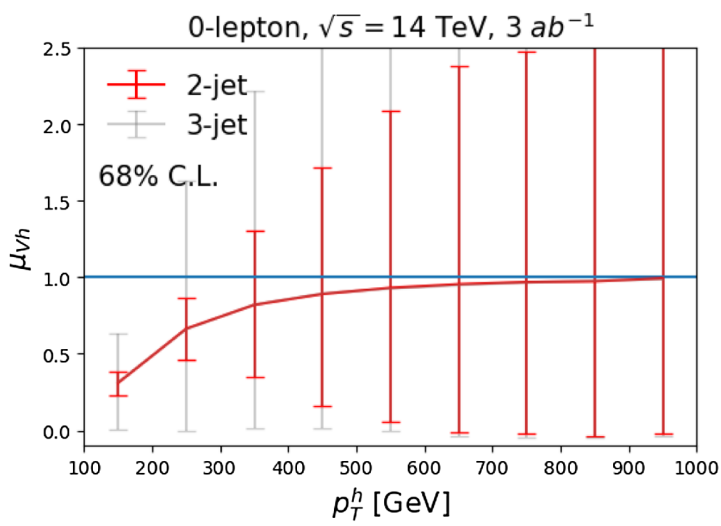

(e)

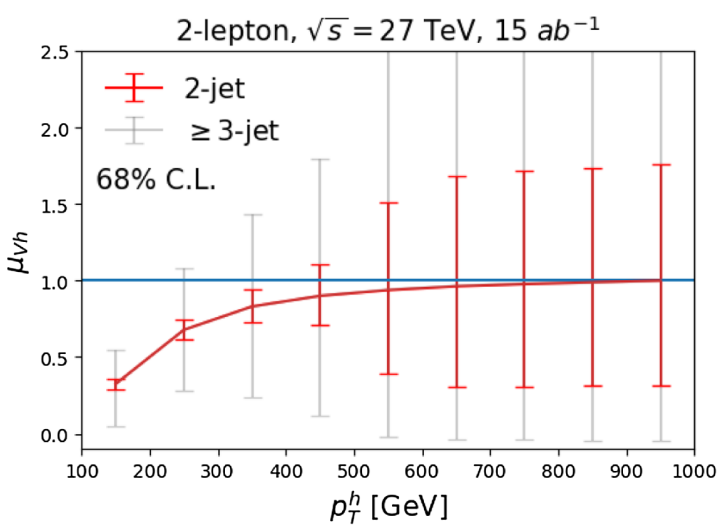

(b)

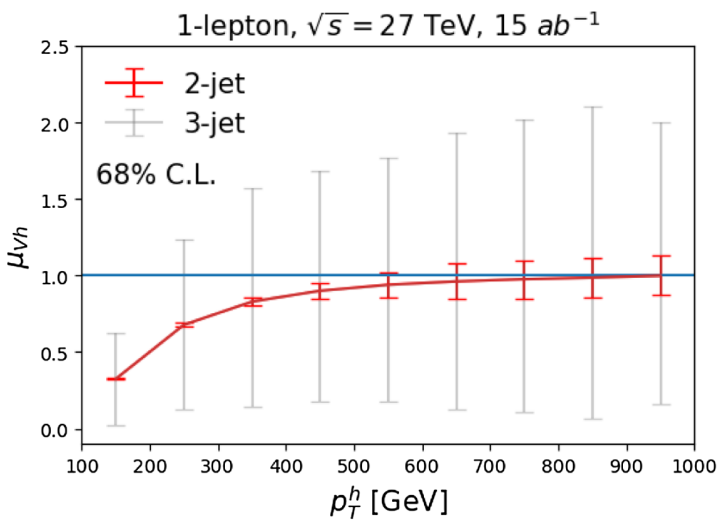

(d)

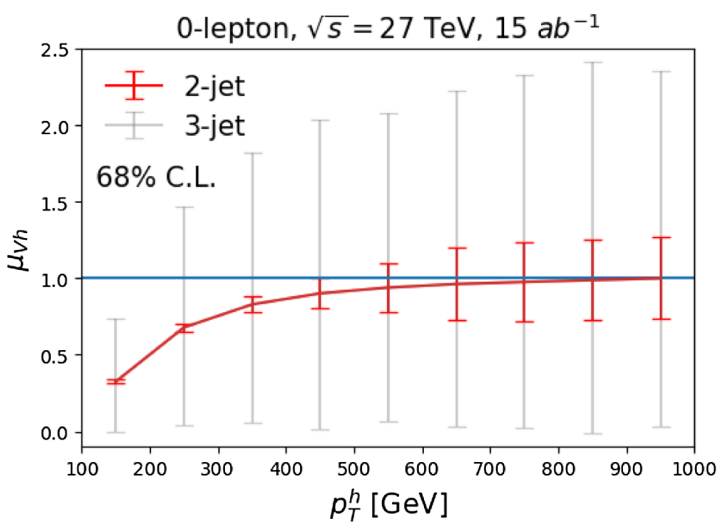

(f)

FIG. 4. Signal strengths $\mu_{V h}$ central values and $68 \%$ CL for (a,b) two lepton categories, (c,d) one lepton categories, and (e,f) zero lepton categories for (a,c,e) $14 \mathrm{TeV}$ with $3 \mathrm{ab}^{-1}$ and (b,d,f) $27 \mathrm{TeV}$ with $15 \mathrm{ab}^{-1}$. We show the (red) two jet and (gray) three jet categories separately.

by using higher and higher energy bins. At low $p_{T}^{h}$ bins, although the statistical error is small, the Goldstone and gauge boson distributions do not agree. As one moves toward higher $p_{T}^{h}$ bins, while the two distributions converge, the statistical errors also increase, as shown in Figs. 4 and 5. In this section we explore statistical measures of the restoration and discuss their implications, taking into account both the theory convergence as well as the experimental uncertainties. The goal is, assuming that the SM is a good description of the data, we want to test the agreement between the $q \bar{q}^{\prime} \rightarrow$ $V h$ and $q \bar{q}^{\prime} \rightarrow G h\left(\mu_{V h}^{j}=1\right)$ production as a function of $p_{T}^{h}$.

As a first choice, using the language that the high energy physics community is more familiar with, we consider using " $\chi^{2}$ per degree of freedom" as a function of $p_{T}$ bins. 


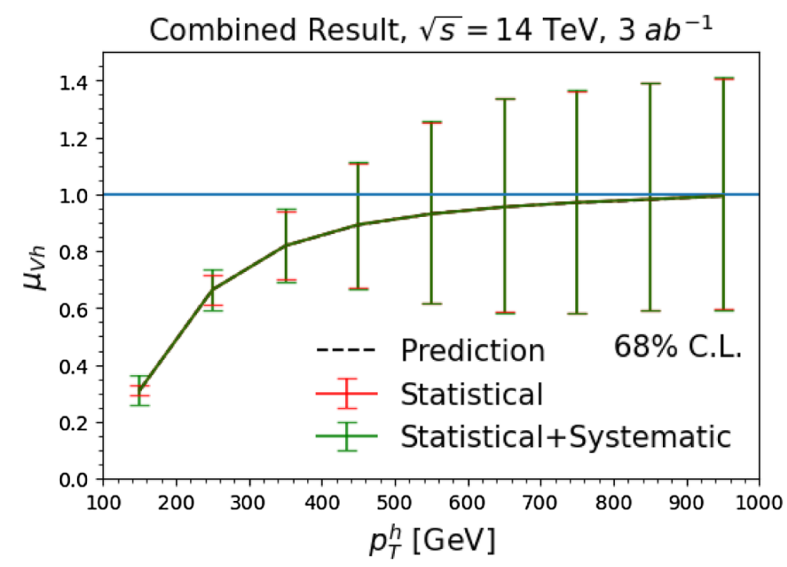

(a)

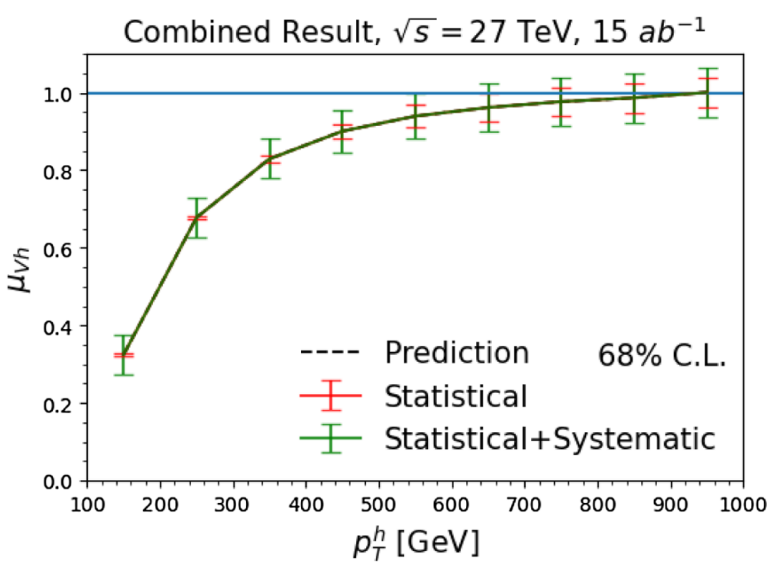

(b)

FIG. 5. Combined central values across all categories and 68\% CL for extracted signal strengths $\mu_{V h}$ at (a) $14 \mathrm{TeV}^{\mathrm{with}} 3 \mathrm{ab}^{-1}$ and (b) $27 \mathrm{TeV}$ with $15 \mathrm{ab}^{-1}$. Black dashed lines are the partonic level prediction, the red bars are statistical uncertainty, the green bars are statistical and a $5 \%$ systematic uncertainty added in quadrature.

One generically anticipates this quantity to decrease as an indicator of better convergence. After using the method in the previous sections in separating signal and background, we now have six-category samples, post-selection cuts, that have the significance of our analysis as a function of $p_{T}^{h}$. One can define " $\chi^{2}$ per degree of freedom"

$\Delta \chi_{m}^{2}=\frac{1}{m} \sum_{l=1}^{m} \log \left(\frac{\operatorname{Pois}\left(n_{o b s, l} \mid \sum_{j} \Delta \sigma_{j}^{G h} \epsilon_{l j} L+B_{l}\right)}{\operatorname{Pois}\left(n_{o b s, l} \mid S_{l}+B_{l}\right)}\right)$,

where we sum over the $m$ ranked $p_{T}^{h}$ bins (from low to high). Using the methods of the previous section, we perform 10,000 pseudoexperiments. The results are shown in the left panel of Fig. 6. We show the median over all pseudoexperiments as well as the band where $68 \%$ and $95 \%$ of the pseudoexperiments lie. From the figure we can see, as anticipated, the $\Delta \chi_{m}^{2}$ decreases as one includes more high $p_{T}^{h}$ bins.

However, we note here that $\Delta \chi_{m}^{2}$ has some disadvantages in measuring restoration. First, for the low $p_{T}^{h}$ bins, each bin contributes to a sizable $\Delta \chi^{2}$ since the $G h$ and $V h$ hypothesis are in poor agreement and statistical uncertainty is small. At high $p_{T}^{h}$, the statistical uncertainties increase. Hence, even if the $G h$ and $V h$ distributions do not converge, as more bins are averaged over $\Delta \chi_{m}^{2}$ will decrease. In other words, even if the higher bins contain no separation power, e.g., the background uncertainty being infinitely larger than the signal strength, the $\Delta \chi_{m}^{2}$ decreases. This reflects that $\Delta \chi_{m}^{2}$ measures the agreement between two hypotheses: as the uncertainties increase, the error bars overlap, and the hypotheses are in "good agreement." However, to measure EW restoration, the

\footnotetext{
${ }^{3}$ Here we use the log-likelihood ratio as delta chi-square for each bin.
}

convergence of $V h$ and $G h$ must be measured and $\Delta \chi_{m}^{2}$ is not a good measure of convergence.

As can be seen, the measurement of the EW restoration is not a typical particle physics test. The issue is that we want to measure the convergence of two hypotheses with energy, not just determine how well they agree globally. Ideally, the measure should contrast different hypotheses for a given experimental data set with proper weight for each bin according to the "information" contained there. We turn to Shannon's information theory and find that generically $-p \log p$ measures the information of a distribution $p$. While there might be an equivalent or better definition outside of our scope, we use a modified Kullback-Leibler (KL) divergence. KL divergence is a commonly used quantity contrasting the information between two different distributions, and often plays the role of loss function for machine learning. The KL divergence tests the information difference between two hypotheses. To do this, for each pseudoexperiment we first define properly normalized probability for each bin for the $V h$ hypothesis

$$
p_{i}^{\leq m}=\prod_{\substack{\text { Gignal } \\ \text { categoies }}} \frac{\operatorname{Pois}\left(n_{o b s, i} \mid S_{i}+B_{i}\right)}{\sum_{l=1}^{m} \operatorname{Pois}\left(n_{o b s, l} \mid S_{l}+B_{l}\right)},
$$

where the $i \leq m$ are bin numbers with increasing $p_{T}^{h}$. We have assumed independent event samples, and so have taken a product of probabilities across all signal categories. Restricting ourselves to $p_{T}^{h} \leq p_{T, m}^{h}$ with $p_{T, m}^{h}$ being the central value of $p_{T}^{h}$ in the $m$ th bin, $p_{i}^{\leq m}$ is the probability of observing $n_{o b s, i}$ events in bin $i$ given a SM hypothesis of $S_{i}+B_{i}$ bins. The $G h$ hypothesis is equivalent to signal strengths of one: $\mu_{V h}=1$. Using the efficiency matrices $\epsilon_{i j}$ we can define an analogue conditional probability for the $G h$ hypothesis: 


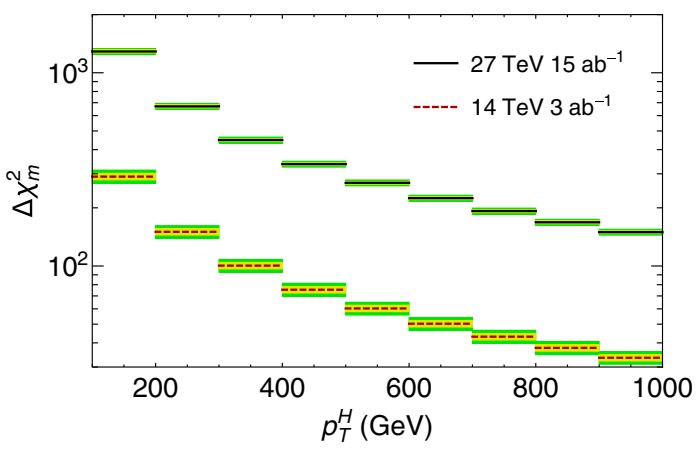

(a)

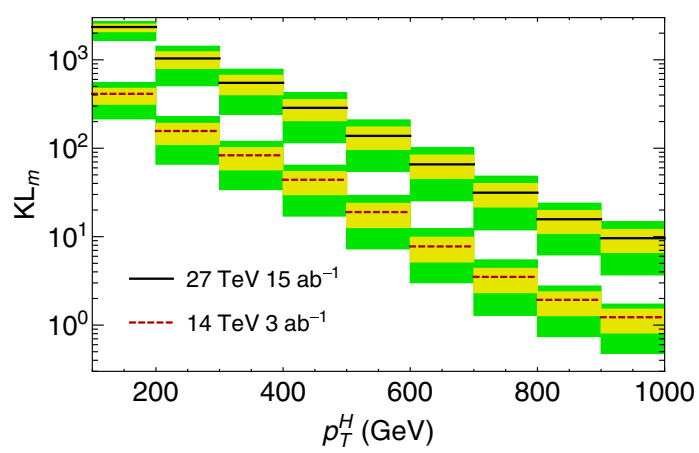

(b)

FIG. 6. The $\chi^{2}$ per degree of freedom $\Delta \chi_{m}^{2}$ defined in Eq. (24) (left panel) KL divergence defined in Eq. (27) (right panel) for 14 TeV with $3 \mathrm{ab}^{-1}$ and $27 \mathrm{TeV}$ with $15 \mathrm{ab}^{-1}$. The back (red dashed) line represents the median values of the $27 \mathrm{TeV}$ (14 TeV) results, and the yellow and green bands represent the values where $68 \%$ and $95 \%$ of pseudoexperiments lie, respectively.

$q_{i}^{\leq m}=\prod_{\substack{6 \text { signal } \\ \text { categories }}} \frac{\operatorname{Pois}\left(n_{o b s, i} \mid \sum_{j} \Delta \sigma_{j}^{G h} \epsilon_{i j} L+B_{i}\right)}{\sum_{l=1}^{m} \operatorname{Pois}\left(n_{o b s, l} \mid \sum_{j} \Delta \sigma_{j}^{G h} \epsilon_{l j} L+B_{l}\right)}$,

where the sum over $j$ is over all bins and not restricted to bins less than $p_{T, m}^{h}$. The KL divergence for the first $m$ bins is then:

$$
K L_{m}=\sum_{i=1}^{m} p_{i}^{\leq m} \log \left(\frac{p_{i}^{\leq m}}{q_{i}^{\leq m}}\right)
$$

Now the interpretation of the KL-divergence is clear. If the two hypotheses describe the data equally well, the log goes to zero and the KL divergence is zero. The KL-divergence has a similar property as the Gibbs free energy, being positive definite. Hence, when the agreement of the two hypotheses is worse, $K L_{m}$ is larger. As more bins are included, we expect the EW restoration to describe data better and the KL divergence should approach zero.

When the two hypotheses do not agree, the weighted sum in Eq. (27) guarantees that the largest contributions come from bins for the conditional probabilities $p_{i}^{\leq m}$ are largest. Hence, the KL divergence contains more information than $\Delta \chi_{m}^{2}$ and is expected to be a better measure of convergence. In Fig. 6 we show the differential KL divergence, $K L_{m}$. We show the median over all pseudoexperiments as well as the band where $68 \%$ and $95 \%$ of the pseudo-experiments lie. As can be clearly seen, whereas the $\chi^{2}$ per degree of freedom test began to plateau at high energies, the KL-divergence decrease more steadily. This more readily shows that the agreement of the $V h$ and $G h$ hypotheses continues to get better at high $p_{T}^{h}$ and we observe EW restoration.

We want to emphasize here that the convergence between $V h$ and $G h$ distributions is directly represented by the fact that $\Delta \chi_{m}^{2}$ and $K L_{m}$ decrease as higher and higher $p_{T}^{h}$ bins are included. We would like to note that somewhat counterintuitively the $14 \mathrm{TeV}$ statistical tests seem to be "better" than the $27 \mathrm{TeV}$ results. That is, the $14 \mathrm{TeV}$ values are lower. Even if we had $p_{T}$ bins larger than $1 \mathrm{TeV}$, the $27 \mathrm{TeV}$ results will not "beat" the $14 \mathrm{TeV}$ results. This is because the smaller uncertainties at $27 \mathrm{TeV}$ cause the first bin, where agreement is poor, to be considerably greater than the $14 \mathrm{TeV}$ results. This results in the entire $27 \mathrm{TeV}$ $\Delta \chi_{m}^{2}$ and $K L_{m}$ distributions being greater than at $14 \mathrm{TeV}$. That is, although the uncertainties of the differential cross sections between different machines can be compared to determine which machine is more sensitive, the nonconventional tests of the convergence of the signal strengths, i.e., $\Delta \chi_{m}^{2}$ and $K L_{m}$, should be considered on a machine-bymachine basis.

\section{CONCLUSIONS}

In this paper, we have studied the potential of the HLLHC and HE-LHC to observe EW restoration in $p p \rightarrow V h$ production. Discussions of EW symmetry restoration have traditionally been limited to the longitudinal vector boson scattering. Using the Goldstone boson equivalence theorem, it can be seen that this scattering occurs via the quartic term in the Higgs potential. However, Goldstone bosons also have interactions via the Higgs kinetic terms. These terms contribute to the production of longitudinal gauge bosons in $q \bar{q}^{\prime} \rightarrow V V^{\prime}$ and $q \bar{q}^{\prime} \rightarrow V h$ channels.

As we showed, the $q \bar{q}^{\prime} \rightarrow V V^{\prime}$ production is dominated by transverse polarizations to very high energies. Hence, it is difficult to observe Goldstone boson production in this channel. Since $q \bar{q}^{\prime} \rightarrow V h$ is a purely $s$-channel process with a component of the Higgs doublet, it is longitudinally dominated starting at relatively low energies. In Sec. II, we defined EW symmetry restoration by taking the limit of the Higgs vev going to zero and enforcing the SM tree-level relations for the Higgs potential parameters. This results in a massless Higgs doublet in the EW restored theory. From this, we defined a differential signal strength $\mu_{V h}$ as the ratio of the $p_{T}^{h}$ distributions of $V h$ and $G h$ production. As shown, this signal strength is the same 
for $Z h$ and $W^{ \pm} h$, allowing for easy extraction of global signal strength in all $V h$ channels. This convergence can be seen in Fig. 2.

As EW symmetry is restored, the longitudinal gauge bosons are replaced with their Goldstone boson counterparts. Hence, the signal strength $\mu_{V h}$ is expected to converge to the Goldstone calculation at high energies. Using a sophisticated collider analysis, we showed that by performing a fit to these signal strengths, it can be observed that the $V h$ channel converges to $G h$ at high energies. Indeed, for $p_{T}^{h} \sim 400 \mathrm{GeV}$, the $V h$ and $G h$ distributions agree at around $80 \%$. Additionally, as can be seen in Figs. 4 and 5, the extracted signal strength in all $V h$ signal categories agrees with the partonic level prediction. Finally, to quantify the agreement between the $V h$ and $G h$ hypothesis, we defined a differential Kullback-Leibler divergence. If two hypotheses agree, the KL divergence is small. As shown in Fig. 6, the $V h$ and $G h$ hypothesis agree well at high energy, and EW restoration can be well observed.

To summarize, we demonstrated the EW restoration could be observed in the $V h$ channel. Indeed, EW restoration can be confirmed to $40 \%$ precision at the HL-LHC and $6 \%$ precision at the HE-LHC. Our study can be further extended to other future colliders, as well as the other diboson production modes highlighted in the theory discussion. Our study clearly highlights the possibility of studying the physics phenomena of electroweak restoration at high energy colliders as well as electroweak breaking.

\section{ACKNOWLEDGMENTS}

I. M. L. would like to thank the Institute for Theoretical Physics at Universität Heidelberg for their hospitality during the beginning of this work and Tilman Plehn for insightful discussions. I. M. L. would also like to thank Sally Dawson for useful comments. This work was performed in part at the Aspen Center for Physics, which is supported by National Science Foundation Grant No. PHY1607611. L. H. and I. M. L. were supported in part in part by United States Department of Energy Grant No. de-sc0017988. S. L. is supported by the State of Kansas EPSCoR grant program and the U.S. Department of Energy, Office of Science, Office of Workforce Development for Teachers and Scientists, Office of Science Graduate Student Research (SCGSR) program. The SCGSR program is administered by the Oak Ridge Institute for Science and Education (ORISE) for the DOE. ORISE is managed by ORAU under Contract No. DESC0014664. Z. L. was supported in part by the NSF Grants No. PHY-1620074, No. PHY-1914480, and No. PHY1914731, and by the Maryland Center for Fundamental Physics (MCFP). The data to reproduce the plots has been uploaded with the arXiv submission or is available upon request.

\section{APPENDIX: DNN INPUTS}

First, note the Higgs is always reconstructed from the two leading bottom tagged jets. In the 2-lepton categories the $Z$ is reconstructed from the two leptons, and in the zero lepton category the $Z$ is reconstructed from the missing transverse energy. Note for the zero lepton category, we only reconstruct the $p_{T}$ of the $Z$. In the 1-lepton case, there is a missing neutrino. Its transverse momentum is assumed to be the missing transverse energy of the event. The neutrino's longitudinal momentum is found by requiring that the neutrino plus lepton system reconstruct the $W$-mass. This leads to a two-fold ambiguity, and we choose the neutrino momentum that is closer to the lepton. Hence, in the 1-lepton case the $W$ is reconstructed. Additionally, we label the leading bottom jet at $b_{0}$, the next to leading bottom jet as $b_{1}$, and the leptons as $\ell_{0}, \ell_{1}$ similarly. For 3 -jet categories, we label the leading non- $b$ jet as $j$.

The following definitions are used:

(i) The invariant mass of two objects $i, j$ is $M_{i j}$.

(ii) The reconstructed mass of an object $i$ is $M_{i}^{\text {recon }}$.

(iii) For a final state system $i$, the transverse mass is defined at $M_{T, i}=\sqrt{E_{i}^{2}-p_{Z, i}^{2}}$, where $E_{i}$ is the total energy of the objects $i$ and $p_{Z, i}$ is the $z$-component of their momentum.

(iv) The transverse momentum of an object $i$ is $p_{T}^{i}$.

(v) The azimuthal difference between two objects $i, j$ is $\Delta \phi_{i j}=\left|\phi_{i}-\phi_{j}\right|$.

(vi) The difference between the rapidities of two objects $i, j$ is $\Delta \eta_{i j}=\left|\eta_{i}-\eta_{j}\right|$.

(vii) The opening angle between two objects $i, j$ is $\left(\Delta R_{i j}\right)^{2}=\left(\Delta \phi_{i j}\right)^{2}+\left(\Delta \eta_{i j}\right)^{2}$.

(viii) The scalar sum of all transverse momentum is $H_{T}$.

(ix) The number of non- $b$ jets is $n_{j}$.

The input variables for the DNN for each event category are

(i) 2-lepton, 2-jet:

- $M_{Z h}, M_{h}^{\text {recon }}, M_{Z}^{\text {recon }}$.

- MET, $p_{T}^{h}, p_{T}^{Z}, p_{T}^{b_{0}}, p_{T}^{b_{1}}, p_{T}^{\ell_{0}}, p_{T}^{\ell_{1}}, H_{T}$.

- $\Delta \phi_{Z h}, \Delta \eta_{Z h}, \Delta R_{Z h}$.

- $\Delta R_{b_{0} b_{1}}, \Delta R_{\ell_{0} \ell_{1}}, \Delta R_{b_{0} \ell_{0}}, \Delta R_{b_{1} \ell_{1}}$.

- Transverse mass of reconstructed Higgs and $\mathrm{Z}, M_{T, Z h}$.

(ii) 2-lepton, 3-jet: Same as 2-lep + 2-jet with:

- $p_{T}^{j}, \Delta R_{h j}, \Delta R_{Z j}$.

$-n_{j}$.

(iii) 1-lepton, 2-jet:

$-M_{W H}, M_{h}^{\text {recon }}, M_{W}^{\text {recon }}$.

- MET, $p_{T}^{h}, p_{T}^{W}, p_{T}^{b_{0}}, p_{T}^{b_{1}}, H_{T}$.

- $\Delta \phi_{W h}, \Delta R_{b 0 b 1}, \min \left\{\Delta \phi_{\ell 0 b 0}, \Delta \phi_{\ell 0 b 1}\right\}$.

- Transverse mass of reconstructed Higgs and $\mathrm{W}, M_{T, W h}$.

- Transverse mass of $W: M_{T, W}$.

- Transverse mass of $W+b_{0}: M_{T, W b_{0}}$. 
- Transverse mass of $W+b_{1}: M_{T, W b_{1}}$.

- $\left|\Delta Y_{h W}\right|=\left|\eta_{h}-\eta_{W}\right|$, where $\eta_{h, W}$ are the Higgs and $W$ rapidities.

(iv) 1-lepton, 3-jet: Same as 1-lepton, 2-jet with:

- $p_{T}^{j}, M_{h j}$

(v) 0-lepton, 2-jet:

$-M_{h}^{\text {recon }}$.
- MET, $p_{T}^{h}, p_{T}^{b_{0}}, p_{T}^{b_{1}}, H_{T}$.

- $\Delta \phi_{Z h}, \Delta \eta_{b_{0} b_{1}}, \Delta R_{b_{0} b_{1}}$.

- Transverse momentum of reconstructed Higgs and Z, $M_{T, Z h}$.

(vi) 0-lepton, 3-jet: Same as 0-lepton, 2-jet with:

$-M_{h j}, p_{T}^{j}$.
[1] G. Aad et al. (ATLAS Collaboration), Observation of a new particle in the search for the Standard Model Higgs boson with the ATLAS detector at the LHC, Phys. Lett. B 716, 1 (2012).

[2] S. Chatrchyan et al. (CMS Collaboration), Observation of a new boson at a mass of $125 \mathrm{GeV}$ with the CMS experiment at the LHC, Phys. Lett. B 716, 30 (2012).

[3] M. Mangano et al., Physics at a $100 \mathrm{TeV}$ pp collider: Standard model processes, CERN Yellow Rep. (CERN, Geneva, 2017), Vol. 3, p. 1.

[4] M. Farina, G. Panico, D. Pappadopulo, J. T. Ruderman, R. Torre, and A. Wulzer, Energy helps accuracy: Electroweak precision tests at hadron colliders, Phys. Lett. B 772, 210 (2017).

[5] C. Englert, R. Rosenfeld, M. Spannowsky, and A. Tonero, New physics and signal-background interference in associated $p p \rightarrow H Z$ production, Europhys. Lett. 114, 31001 (2016).

[6] D. R. Green, P. Meade, and M.-A. Pleier, Multiboson interactions at the LHC, Rev. Mod. Phys. 89, 035008 (2017).

[7] A. Butter, O. J. P. Éboli, J. Gonzalez-Fraile, M. C. GonzalezGarcia, T. Plehn, and M. Rauch, The gauge-Higgs legacy of the LHC Run I, J. High Energy Phys. 07 (2016) 152.

[8] Z. Zhang, Time to Go Beyond Triple-Gauge-BosonCoupling Interpretation of $W$ Pair Production, Phys. Rev. Lett. 118, 011803 (2017).

[9] R. Franceschini, G. Panico, A. Pomarol, F. Riva, and A. Wulzer, Electroweak precision tests in high-energy Diboson processes, J. High Energy Phys. 02 (2018) 111.

[10] I. Brivio and M. Trott, The standard model as an effective field theory, Phys. Rep. 793, 1 (2019).

[11] J. Baglio, S. Dawson, and I. M. Lewis, An NLO QCD effective field theory analysis of $W^{+} W^{-}$production at the LHC including fermionic operators, Phys. Rev. D 96, 073003 (2017).

[12] D. Liu and L.-T. Wang, Prospects for precision measurement of Diboson processes in the semileptonic decay channel in future LHC runs, Phys. Rev. D 99, 055001 (2019).

[13] J. Baglio, S. Dawson, and I. M. Lewis, NLO effects in EFT fits to $W^{+} W^{-}$production at the LHC, Phys. Rev. D 99, 035029 (2019).

[14] E. da Silva Almeida, A. Alves, N. Rosa Agostinho, O. J. P. Éboli, and M.C. Gonzalez-Garcia, Electroweak aector under scrutiny: A combined analysis of LHC and electroweak precision data, Phys. Rev. D 99, 033001 (2019).

[15] J. Ellis, C. W. Murphy, V. Sanz, and T. You, Updated global SMEFT fit to Higgs, Diboson and electroweak data, J. High Energy Phys. 06 (2018) 146.

[16] A. Biekötter, D. Gonçalves, T. Plehn, M. Takeuchi, and D. Zerwas, The global Higgs picture at $27 \mathrm{TeV}$, SciPost Phys. 6, 024 (2019).

[17] A. Alves, N. Rosa-Agostinho, O. J. P. Éboli, and M. C. Gonzalez-Garcia, Effect of fermionic operators on the gauge legacy of the LHC run I, Phys. Rev. D 98, 013006 (2018).

[18] L. Di Luzio, R. Gröber, and G. Panico, Probing new electroweak states via precision measurements at the LHC and future colliders, J. High Energy Phys. 01 (2019) 011.

[19] S. Banerjee, C. Englert, R. S. Gupta, and M. Spannowsky, Probing electroweak precision physics via boosted Higgs-Strahlung at the LHC, Phys. Rev. D 98, 095012 (2018).

[20] C. Hays, A. Martin, V. Sanz, and J. Setford, On the impact of dimension-eight SMEFT operators on Higgs measurements, J. High Energy Phys. 02 (2019) 123.

[21] A. Biekoetter, T. Corbett, and T. Plehn, The gauge-Higgs legacy of the LHC run II, SciPost Phys. 6, 064 (2019).

[22] C. Grojean, M. Montull, and M. Riembau, Diboson at the LHC vs LEP, J. High Energy Phys. 03 (2019) 020.

[23] B. Henning, D. M. Lombardo, and F. Riva, Improved BSM sensitivity in Diboson processes at linear colliders, Eur. Phys. J. C 80, 220 (2020).

[24] J. De Blas, G. Durieux, C. Grojean, J. Gu, and A. Paul, On the future of Higgs, electroweak and diboson measurements at lepton colliders, J. High Energy Phys. 12 (2019) 117.

[25] S. Banerjee, R. S. Gupta, J. Y. Reiness, S. Seth, and M. Spannowsky, Towards the ultimate differential SMEFT analysis, J. High Energy Phys. 09 (2020) 170.

[26] G. Cuomo, L. Vecchi, and A. Wulzer, Goldstone equivalence and high energy electroweak physics, SciPost Phys. 8, 078 (2020).

[27] W. H. Chiu, Z. Liu, and L.-T. Wang, Probing flavor nonuniversal theories through Higgs physics at the LHC and future colliders, Phys. Rev. D 101, 035045 (2020).

[28] J. Brehmer, S. Dawson, S. Homiller, F. Kling, and T. Plehn, Benchmarking simplified template cross sections in $\mathrm{WH}$ production, J. High Energy Phys. 11 (2019) 034. 
[29] P. Azzi et al., Report from working group 1: Standard model physics at the HL-LHC and HE-LHC, CERN Yellow Rep. Monogr. (CERN, Geneva, 2019), Vol. 7, p. 1.

[30] J. Baglio, S. Dawson, and S. Homiller, QCD corrections in standard model EFT fits to $W Z$ and $W W$ production, Phys. Rev. D 100, 113010 (2019).

[31] A. Delgado and A. Martin, Reinterpreting $p p \rightarrow W^{+} W^{-}$ searches for charginos, Phys. Rev. D 101, 035014 (2020).

[32] F. F. Freitas, C. K. Khosa, and V. Sanz, Exploring the standard model EFT in VH production with machine learning, Phys. Rev. D 100, 035040 (2019).

[33] F. Bishara, P. Englert, C. Grojean, M. Montull, G. Panico, and A. N. Rossia, A new precision process at FCC-hh: The diphoton leptonic Wh channel, J. High Energy Phys. 07 (2020) 075.

[34] L. Ricci, R. Torre, and A. Wulzer, On the W\&Y interpretation of high-energy Drell-Yan measurements, arXiv: 2008.12978.

[35] F. Bishara, S. De Curtis, L. Delle Rose, P. Englert, C. Grojean, M. Montull, G. Panico, and A. N. Rossia, Precision from the diphoton $\mathrm{Zh}$ channel at FCC-hh, arXiv:2011.13941.

[36] J. Y. Araz, S. Banerjee, R. S. Gupta, and M. Spannowsky, Precision SMEFT bounds from the VBF Higgs at high transverse momentum, arXiv:2011.03555.

[37] J. Baglio, S. Dawson, S. Homiller, S. D. Lane, and I. M. Lewis, Validity of standard model EFT studies of VH and VV production at NLO, Phys. Rev. D 101, 115004 (2020).

[38] S. Dawson, S. Homiller, and S. D. Lane, Putting standard model EFT fits to work, Phys. Rev. D 102, 055012 (2020).

[39] J. Ellis, M. Madigan, K. Mimasu, V. Sanz, and T. You, Top, Higgs, Diboson and electroweak fit to the standard model effective field theory, arXiv:2012.02779.

[40] R. Aoude, T. Hurth, S. Renner, and W. Shepherd, The impact of flavour data on global fits of the MFV SMEFT, J. High Energy Phys. 12 (2020) 113.

[41] S. Dawson, The effective W approximation, Nucl. Phys. B249, 42 (1985).

[42] G. L. Kane, W. Repko, and W. Rolnick, The effective $W+-$, Z0 approximation for high-energy collisions, Phys. Lett. 148B, 367 (1984).

[43] Z. Kunszt and D. E. Soper, On the validity of the effective $W$ approximation, Nucl. Phys. B296, 253 (1988).

[44] C. W. Bauer, N. Ferland, and B. R. Webber, Standard model parton distributions at very high energies, J. High Energy Phys. 08 (2017) 036.

[45] C. W. Bauer and B. R. Webber, Polarization effects in standard model parton distributions at very high energies, J. High Energy Phys. 03 (2019) 013.

[46] T. Han, Y. Ma, and K. Xie, High energy leptonic collisions and electroweak parton distribution functions, Phys. Rev. D 103, L031301 (2021).

[47] A. Hook and A. Katz, Unbroken $S U(2)$ at a $100 \mathrm{TeV}$ collider, J. High Energy Phys. 09 (2014) 175.

[48] J. Chen, T. Han, and B. Tweedie, Electroweak splitting functions and high energy showering, J. High Energy Phys. 11 (2017) 093.

[49] C. W. Bauer, D. Provasoli, and B. R. Webber, Standard model fragmentation functions at very high energies, J. High Energy Phys. 11 (2018) 030.
[50] L. Rinchiuso, O. Macias, E. Moulin, N. L. Rodd, and T. R. Slatyer, Prospects for heavy WIMP dark matter with CTA: The Wino and Higgsino, Phys. Rev. D 103, 023011 (2021).

[51] C. H. Llewellyn Smith, High-energy behavior and gauge symmetry, Phys. Lett. 46B, 233 (1973).

[52] M. J. G. Veltman, Second threshold in weak interactions, Acta Phys. Pol. B 8, 475 (1977), https://www.actaphys.uj .edu.pl/R/8/6/475/pdf.

[53] B. W. Lee, C. Quigg, and H. B. Thacker, Weak interactions at very high-energies: The role of the Higgs boson mass, Phys. Rev. D 16, 1519 (1977).

[54] M. S. Chanowitz and M. K. Gaillard, The TeV physics of strongly interacting W's and Z's, Nucl. Phys. B261, 379 (1985).

[55] D. A. Dicus, J.F. Gunion, and R. Vega, Isolating the scattering of longitudinal W+'s at the SSC using like sign dileptons, Phys. Lett. B 258, 475 (1991).

[56] V. D. Barger, K.-m. Cheung, T. Han, and R. J. N. Phillips, Strong $W^{+} W^{+}$scattering signals at $p p$ supercolliders, Phys. Rev. D 42, 3052 (1990).

[57] J. Bagger, S. Dawson, and G. Valencia, Effective field theory of anomalous gauge-boson couplings at highenergy pp colliders, Nucl. Phys. B399, 364 (1993).

[58] D. A. Dicus and V. S. Mathur, Upper bounds on the values of masses in unified gauge theories, Phys. Rev. D 7, 3111 (1973).

[59] J. Bagger, V. D. Barger, K.-m. Cheung, J. F. Gunion, T. Han, G. A. Ladinsky, R. Rosenfeld, and C. P. Yuan, The strongly interacting W W system: Gold plated modes, Phys. Rev. D 49, 1246 (1994).

[60] J. Bagger, V. D. Barger, K.-m. Cheung, J. F. Gunion, T. Han, G. A. Ladinsky, R. Rosenfeld, and C. P. Yuan, CERN LHC analysis of the strongly interacting W W system: Gold plated modes, Phys. Rev. D 52, 3878 (1995).

[61] M. S. Chanowitz, Strong W W scattering at the end of the 90's: Theory and experimental prospects, in Hidden symmetries and Higgs phenomena. Proceedings, Summer School, Zuoz, Switzerland (Paul Scherrer Inst., VilligenSchwenningen, 1998), pp. 81-109.

[62] J. M. Butterworth, B. E. Cox, and J. R. Forshaw, $W W$ scattering at the CERN LHC, Phys. Rev. D 65, 096014 (2002).

[63] T. Han, D. Krohn, L.-T. Wang, and W. Zhu, New physics signals in longitudinal gauge boson scattering at the LHC, J. High Energy Phys. 03 (2010) 082.

[64] J. Brehmer, J. Jaeckel, and T. Plehn, Polarized WW scattering on the Higgs pole, Phys. Rev. D 90, 054023 (2014).

[65] G. Aad et al. (ATLAS and CMS Collaborations), Measurements of the Higgs boson production and decay rates and constraints on its couplings from a combined ATLAS and CMS analysis of the LHC pp collision data at $\sqrt{\mathrm{s}}=7$ and $8 \mathrm{TeV}$, J. High Energy Phys. 08 (2016) 045.

[66] D. de Florian et al. (LHC Higgs Cross Section Working Group), Handbook of LHC Higgs Cross Sections: 4. Deciphering the Nature of the Higgs Sector, arXiv: 1610.07922.

[67] A. M. Sirunyan et al. (CMS Collaboration), Combined measurements of Higgs boson couplings in proton-proton collisions at $\sqrt{s}=13 \mathrm{TeV}$, Eur. Phys. J. C 79, 421 (2019). 
[68] G. Aad et al. (ATLAS Collaboration), Combined measurements of Higgs boson production and decay using up to $80 \mathrm{fb}^{-1}$ of proton-proton collision data at $\sqrt{s}=13 \mathrm{TeV}$ collected with the ATLAS experiment, Phys. Rev. D 101, 012002 (2020).

[69] A. Belyaev, A. Oliveira, R. Rosenfeld, and M. C. Thomas, Multi Higgs and vector boson production beyond the standard model, J. High Energy Phys. 05 (2013) 005.

[70] T. Corbett, O. Éboli, and M. Gonzalez-Garcia, Unitarity constraints on dimension-six operators II: Including fermionic operators, Phys. Rev. D 96, 035006 (2017).

[71] A. Ballestrero, E. Maina, and G. Pelliccioli, $W$ boson polarization in vector boson scattering at the LHC, J. High Energy Phys. 03 (2018) 170.

[72] A. Falkowski and R. Rattazzi, Which EFT, J. High Energy Phys. 10 (2019) 255.

[73] S. Chang and M. A. Luty, The Higgs trilinear coupling and the scale of new physics, J. High Energy Phys. 03 (2020) 140.

[74] J. Baglio and N. Le Duc, Fiducial polarization observables in hadronic WZ production: A next-to-leading order QCD + EW study, J. High Energy Phys. 04 (2019) 065.

[75] A. Denner and G. Pelliccioli, Polarized electroweak bosons in $\mathrm{W}^{+} \mathrm{W}^{-}$production at the LHC including NLO QCD effects, J. High Energy Phys. 09 (2020) 164.

[76] K. Gaemers and G. Gounaris, Polarization amplitudes for $e+e-\rightarrow W+W-$ and $e+e-\rightarrow \mathrm{ZZ}$, Z. Phys. C 1, 259 (1979).

[77] M. J. Duncan, G. L. Kane, and W. Repko, W W physics at future colliders, Nucl. Phys. B272, 517 (1986).

[78] K. Hagiwara, R. Peccei, D. Zeppenfeld, and K. Hikasa, Probing the weak boson sector in $e+e-\rightarrow W+W-$, Nucl. Phys. B282, 253 (1987).

[79] J. Pumplin, D. Stump, J. Huston, H. Lai, P. M. Nadolsky, and W. Tung, New generation of parton distributions with uncertainties from global QCD analysis, J. High Energy Phys. 07 (2002) 012.

[80] A. Buckley, J. Ferrando, S. Lloyd, K. Nordström, B. Page, M. Rüfenacht, M. Schönherr, and G. Watt, LHAPDF6: Parton density access in the LHC precision era, Eur. Phys. J. C 75, 132 (2015).

[81] D. Clark, E. Godat, and F. Olness, ManeParse: A Mathematica reader for parton distribution functions, Comput. Phys. Commun. 216, 126 (2017).

[82] A. Azatov, J. Elias-Miro, Y. Reyimuaji, and E. Venturini, Novel measurements of anomalous triple gauge couplings for the LHC, J. High Energy Phys. 10 (2017) 027.

[83] G. Panico, F. Riva, and A. Wulzer, Diboson interference resurrection, Phys. Lett. B 776, 473 (2018).

[84] R. Aoude and W. Shepherd, Jet substructure measurements of interference in non-interfering SMEFT effects, J. High Energy Phys. 08 (2019) 009.

[85] S. De, V. Rentala, and W. Shepherd, Measuring the polarization of boosted, hadronic $W$ bosons with jet substructure observables, arXiv:2008.04318.

[86] M. Grossi, J. Novak, D. Rebuzzi, and B. Kersevan, Comparing traditional and deep-learning techniques of kinematic reconstruction for polarisation discrimination in vector boson scattering, Eur. Phys. J. C 80, 1144 (2020).

[87] T. Kim and A. Martin, A $W^{ \pm}$polarization analyzer from deep neural networks, arXiv:2102.05124.
[88] G. Cowan, A survey of unfolding methods for particle physics, Conf. Proc. C0203181, 248 (2002), http:// www.ippp.dur.ac.uk/Workshops/02/statistics/proceedings// cowan.pdf.

[89] Proceedings, PHYSTAT 2011 Workshop on Statistical Issues Related to Discovery Claims in Search Experiments and Unfolding, CERN,Geneva, Switzerland, edited by H. B. Prosper and L. Lyons, CERN Yellow Reports: Conference Proceedings (CERN, Geneva, 2011).

[90] V. Blobel, Unfolding (John Wiley \& Sons, Hoboken, NJ, 2013), pp. 187-225, 10.1002/9783527653416.ch6.

[91] N. D. Gagunashvili, Machine learning approach to inverse problem and unfolding procedure, arXiv:1004.2006.

[92] A. Glazov, Machine learning as an instrument for data unfolding, arXiv:1712.01814.

[93] K. Datta, D. Kar, and D. Roy, Unfolding with generative adversarial networks, arXiv:1806.00433.

[94] A. Andreassen, P. T. Komiske, E. M. Metodiev, B. Nachman, and J. Thaler, OmniFold: A Method to Simultaneously Unfold All Observables, Phys. Rev. Lett. 124, 182001 (2020).

[95] A. M. Sirunyan et al. (CMS Collaboration), Measurement of inclusive and differential Higgs boson production cross sections in the diphoton decay channel in proton-proton collisions at $\sqrt{s}=13 \mathrm{TeV}$, J. High Energy Phys. 01 (2019) 183.

[96] J. Alwall, R. Frederix, S. Frixione, V. Hirschi, F. Maltoni, O. Mattelaer, H. S. Shao, T. Stelzer, P. Torrielli, and M. Zaro, The automated computation of tree-level and nextto-leading order differential cross sections, and their matching to parton shower simulations, J. High Energy Phys. 07 (2014) 079.

[97] T. Sjöstrand, S. Ask, J. R. Christiansen, R. Corke, N. Desai, P. Ilten, S. Mrenna, S. Prestel, C. O. Rasmussen, and P. Z. Skands, An introduction to PYTHiA8.2, Comput. Phys. Commun. 191, 159 (2015).

[98] J. de Favereau, C. Delaere, P. Demin, A. Giammanco, V. Lemaître, A. Mertens, and M. Selvaggi (DELPHES 3 Collaboration), DELPHES3, A modular framework for fast simulation of a generic collider experiment, J. High Energy Phys. 02 (2014) 057.

[99] M. L. Mangano, M. Moretti, F. Piccinini, R. Pittau, and A. D. Polosa, ALPGEN, A generator for hard multiparton processes in hadronic collisions, J. High Energy Phys. 07 (2003) 001.

[100] M. Aaboud et al. (ATLAS Collaboration), Evidence for the $H \rightarrow b \bar{b}$ decay with the ATLAS detector, J. High Energy Phys. 12 (2017) 024.

[101] M. Cepeda et al., Report from Working Group 2: Higgs Physics at the HL-LHC and HE-LHC(CERN, Geneva, 2019), Vol. 7, pp. 221-584, 10.23731/CYRM-2019-007.221.

[102] M. Aaboud et al. (ATLAS Collaboration), Observation of $H \rightarrow b \bar{b}$ decays and $V H$ production with the ATLAS detector, Phys. Lett. B 786, 59 (2018).

[103] A. L. Maas, A. Y. Hannun, and A. Y. Ng, Rectifier nonlinearities improve neural network acoustic models, in ICML Workshop on Deep Learning for Audio, Speech and Language Processing (JMLR.org, Atlanta, 2013), https:// sites.google.com/site/deeplearningicml2013/accepted_ papers. 